ARTICLE

Received 4 Oct 2016 | Accepted 31 Mar 2017 | Published 18 May 2017

DOl: $10.1038 /$ ncomms15482

OPEN

\title{
Selective BET bromodomain inhibition as an antifungal therapeutic strategy
}

Flore Mietton ${ }^{1,2, \star}$, Elena Ferri ${ }^{3, \star}$, Morgane Champleboux ${ }^{1, \star}$, Ninon Zala ${ }^{1}$, Danièle Maubon ${ }^{4,5}$, Yingsheng Zhou ${ }^{3}$, Mike Harbut ${ }^{6}$, Didier Spittler ${ }^{2}$, Cécile Garnaud ${ }^{4,5}$, Marie Courçon ${ }^{1}$, Murielle Chauvel ${ }^{7}$, Christophe d'Enfert ${ }^{7}$, Boris A. Kashemirov ${ }^{3}$, Mitchell Hull ${ }^{6}$, Muriel Cornet ${ }^{4,5}$, Charles E. McKenna ${ }^{3}$, Jérôme Govin ${ }^{1}$ \& Carlo Petosa ${ }^{2}$

Invasive fungal infections cause significant morbidity and mortality among immunocompromised individuals, posing an urgent need for new antifungal therapeutic strategies. Here we investigate a chromatin-interacting module, the bromodomain (BD) from the BET family of proteins, as a potential antifungal target in Candida albicans, a major human fungal pathogen. We show that the BET protein Bdf1 is essential in C. albicans and that mutations inactivating its two BDs result in a loss of viability in vitro and decreased virulence in mice. We report small-molecule compounds that inhibit C. albicans Bdf1 with high selectivity over human BDs. Crystal structures of the Bdf1 BDs reveal binding modes for these inhibitors that are sterically incompatible with the human BET-binding pockets. Furthermore, we report a dibenzothiazepinone compound that phenocopies the effects of a Bdf1 BD-inactivating mutation on C. albicans viability. These findings establish BET inhibition as a promising antifungal therapeutic strategy and identify Bdf1 as an antifungal drug target that can be selectively inhibited without antagonizing human BET function.

\footnotetext{
${ }^{1}$ Institut de Biosciences et Biotechnologies de Grenoble, Laboratoire Biologie à Grande Échelle, Université de Grenoble Alpes, CEA, Inserm, 38000 Grenoble, France. ${ }^{2}$ Institut de Biologie Structurale (IBS), Université de Grenoble Alpes, CEA, CNRS, 38044 Grenoble, France. ${ }^{3}$ Department of Chemistry, Dana and David Dornsife College of Letters, Arts and Sciences, University of Southern California, University Park Campus, Los Angeles, California 90089, USA.

${ }^{4}$ Laboratoire de Parasitologie-Mycologie, Institut de Biologie et Pathologie, CHU Grenoble Alpes, 38043 Grenoble, France. ${ }^{5}$ Laboratoire TIMC-IMAGTheREx, UMR 5525 CNRS, Université Grenoble Alpes, 38058 Grenoble, France. ${ }^{6}$ California Institute for Biomedical Research, 11119 N Torrey Pines Rd, La Jolla, California 92037, USA. ${ }^{7}$ Unité Biologie et Pathogénicité Fongiques, Institut Pasteur, INRA, 75015 Paris, France. * These authors contributed equally to this work. Correspondence and requests for materials should be addressed to C.E.M. (email: mckenna@usc.edu) or to J.G. (email: jerome.govin@inserm.fr) or to C.P. (email: carlo.petosa@ibs.fr).
} 
nvasive fungal infections are a major global health concern, with $\sim 2$ million cases and $>800,000$ deaths estimated annually worldwide ${ }^{1}$. Candida species such as $C$. albicans and C. glabrata are among the most significant human fungal pathogens, with invasive candidiasis yielding $30-40 \%$ mortality ${ }^{2,3}$. An increase in drug-resistant fungal strains and the limited repertoire of available drugs has led to an urgent need for novel therapeutic agents ${ }^{1,4-6}$. Promising results have emerged from the study of chromatin-interacting proteins as antifungal targets, including histone acetyltransferases and deacetylases ${ }^{7,8}$. Histone deacetylase inhibitors have weak antifungal activity when used alone but synergize with antifungal drugs such as azoles and echinocandins ${ }^{8,9}$. Deletion of either the histone deacetylase (HST3) or histone acetyltransferase (RTT109) regulating histone $\mathrm{H} 3$ Lys56 acetylation (H3K56ac) in C. albicans increases susceptibility to genotoxic and antifungal agents ${ }^{10}$. In a study of the Mediator complex subunit Med15, which interacts via its KIX domain with a transcription factor (Pdr1) implicated in pleiotropic drug resistance in C. glabrata, drug-resistant strains were re-sensitized to antifungals by a small-molecule inhibitor targeting the KIX domain-Pdr1 interface ${ }^{11}$. These findings point to an important role of chromatin-interacting proteins in fungal drug susceptibility.

Here we investigated an epigenetic reader module, the bromodomain (BD) from the bromo- and extra-terminal domain (BET) family, as a potential antifungal target in C. albicans. BET proteins are chromatin-associated factors that regulate transcription and chromatin remodelling ${ }^{12}$. Human BET family members are $\operatorname{Brd} 2, \operatorname{Brd} 3, \operatorname{Brd} 4$ and Brdt. BET proteins bind chromatin through their two BDs (BD1 and BD2), which specifically recognize histones acetylated on lysine residues. Whereas canonical BDs bind mono-acetylated histone peptides, BET BDs possess a wider ligand-binding pocket allowing them to recognize diacetylated peptides ${ }^{13,14}$. Small-molecule inhibitors, such as JQ1 and IBET, which selectively target BET BDs have been used to validate BET inhibition as a therapeutic strategy against cancer, cardiovascular disease, inflammatory disorders and other medical conditions, with several inhibitors currently in clinical trials ${ }^{12,15-21}$.

The fungal BET protein Bdf1 has been characterized as a global transcriptional regulator in Saccharomyces cerevisiae, where it regulates over 500 genes $^{22}$. S. cerevisiae Bdf1 (ScBdf1) associates with acetylated histones $\mathrm{H} 3$ and $\mathrm{H} 4$ (refs 22-24) and with the general transcription factor TFIID ${ }^{25}$, and is a subunit of the SWR1 chromatin remodelling complex $24,26,27$. ScBdf1 is also important for chromatin compaction during sporulation ${ }^{28}$ and for the salt stress response ${ }^{29}$. In addition to $B D F 1, S$. cerevisiae possesses a second BET gene, $B D F 2$, which is partly functionally redundant with $B D F 1$ (refs 24,25,30,31). Disruption of BDF1 causes severe morphological and growth defects, while deletion of both $B D F 1$ and $B D F 2$ is lethal ${ }^{22,23}$. Point mutations that abolish ligand binding by $S c B d f 1$ BD1 and BD2 cause growth and sporulation defects ${ }^{24}$ and affect the majority of transcripts altered by disruption of the entire gene ${ }^{22}$. Many pathogenic fungal species (including C. albicans, C. glabrata, Aspergillus fumigatus, Cryptococcus neoformans and Pneumocystis jirovecii) lack BDF2, suggesting that inhibition of the sole BET family protein Bdf1 might significantly reduce viability and virulence ${ }^{32}$.

Here we demonstrate that Bdf1 BD functionality is essential in C. albicans and identify small-molecule inhibitors that target Bdf1 BDs without inhibiting human BET proteins, establishing Bdf1 inhibition as a potential antifungal therapeutic strategy.

\section{Results}

C. albicans Bdf1 BDs bind multi-acetylated histone tails. A phylogenetic tree of human and fungal BET proteins is shown in Fig. 1a. The BDs from C. albicans Bdf1 (CaBdf1) share 31-46\% sequence identity with human BET BDs and 58-66\% identity with those from $S c B d f 1$ and hence are likely to bind multiacetylated $\mathrm{H} 3$ and $\mathrm{H} 4$ tails (Fig. 1b). To verify this hypothesis, we purified recombinant $\mathrm{CaBdf1} \mathrm{BD} 1$ and $\mathrm{BD} 2$ and assessed binding to a microarray of human histone tail peptides bearing diverse post-translational modifications. While $\mathrm{H} 2 \mathrm{~A}$ and $\mathrm{H} 2 \mathrm{~B}$ tail sequences differ considerably between human and C. albicans, those of $\mathrm{H} 3$ and $\mathrm{H} 4$ are nearly perfectly conserved, justifying use of the array (Fig. 1c). As expected, CaBdf1 BDs showed weak binding to mono-acetylated peptides and stronger binding to peptides bearing two or more acetylation marks (Fig. 1d,e and Supplementary Data 1). Of the two BDs, BD1 exhibited more promiscuous binding, recognizing 14 distinct $\mathrm{H} 3$ and $\mathrm{H} 4$ acetylation patterns, versus only 7 for BD2. We replaced a conserved tyrosine residue in the binding pocket of each $\mathrm{BD}$ by phenylalanine, a mutation known to compromise ligand binding in ScBdf1 (refs 22,23) (Supplementary Fig. 1a,b). These two 'YF' mutations (Y248F and Y425F) abolished binding to all acetylated peptides, confirming interaction specificity (Fig. 1d,e). For both $\mathrm{BD} 1$ and BD2, the strongest binding was observed with an $\mathrm{H} 4$ peptide tetra-acetylated on lysines 5, 8, 12 and 16 (hereafter denoted H4ac4). A pull-down assay confirmed H4ac4 peptide recognition by both $\mathrm{BDs}$, which was abolished by the YF mutation (Fig. 1f). Interestingly, $\mathrm{CaBdf1} \mathrm{BD} 1$ and $\mathrm{BD} 2$ bound tetra-acetylated $\mathrm{H} 4$ peptides with comparable strength (Fig. 1e), in contrast with mammalian Brd2, Brd4 and Brdt, which bind tetra-acetylated $\mathrm{H} 4$ more tightly through $\mathrm{BD} 1$ than through $\mathrm{BD} 2$ (refs 13,14), highlighting a certain redundancy in the ligandbinding activity of the two CaBdf1 BDs.

Bdf1 BD functionality is essential in C. albicans. We next asked whether Bdf1 BD function is important for the viability of C. albicans. Although a heterozygous BDF1 deletion mutant generated in strain SN152 (derived from SC5314) exhibited no significant phenotype, we were unable to obtain a homozygous $b d f 1 \Delta / \Delta$ mutant, suggesting that $B D F 1$ is essential. To confirm essentiality we placed the remaining allele of the $B D F 1$ gene in the heterozygous strain under the control of a conditional promoter and evaluated survival under repressive conditions. We used either a methionine-repressible promoter or a newly engineered tetracycline (Tet)-regulatable cassette compatible with animal studies. Tet-dependent gene expression in C. albicans is usually achieved by integrating a chimeric transactivator protein and a Tet-responsive promoter independently into the genome $e^{33,34}$. Here we constructed a cassette allowing integration of all required components in a single step. The cassette contains the transactivator (TetR-VP16), a selective marker (ARG4) and seven tandem Tet-operator elements, which we inserted upstream of the $B D F 1$ open reading frame (ORF) to generate strain $\triangle b d f 1 / p$ TetO-BDF1 (Fig. 2a). Immunoblotting with a polyclonal antibody developed in this study to allow specific CaBdf1 detection (Supplementary Fig. 1c,d) confirmed that Bdf1 was expressed from the pTetO promoter in the absence of doxycycline (Dox), albeit at a weaker level than from the endogenous $B D F 1$ promoter, and was effectively repressed in the presence of Dox (Fig. 2b). Strikingly, the growth of strain $\Delta b d f 1 /$ pTetO-BDF1 mirrored these expression levels: compared to wild type (WT), growth was reduced in the absence of Dox and abrogated in its presence (Fig. 2c). The phenotype was rescued by re-introducing a functional copy of $B D F 1$ (strain $B D F 1-R / p T e t O-$ $B D F 1$ ), confirming that $B D F 1$ is essential in C. albicans.

To verify the importance of $\mathrm{BD}$ function for fungal growth, we generated strains in which one or both Bdf1 BDs were inactivated by domain deletion or by the YF point mutation while the other 


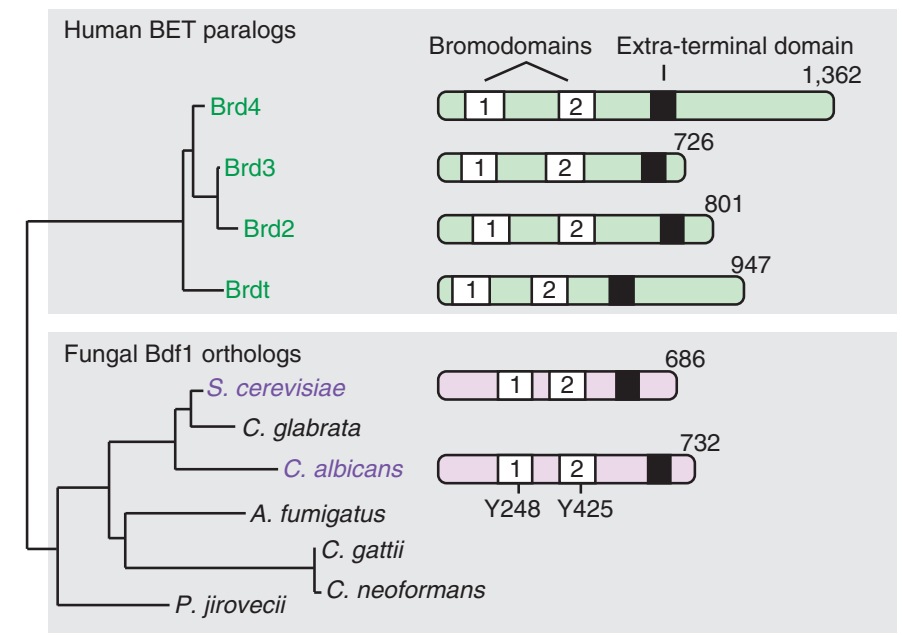

d
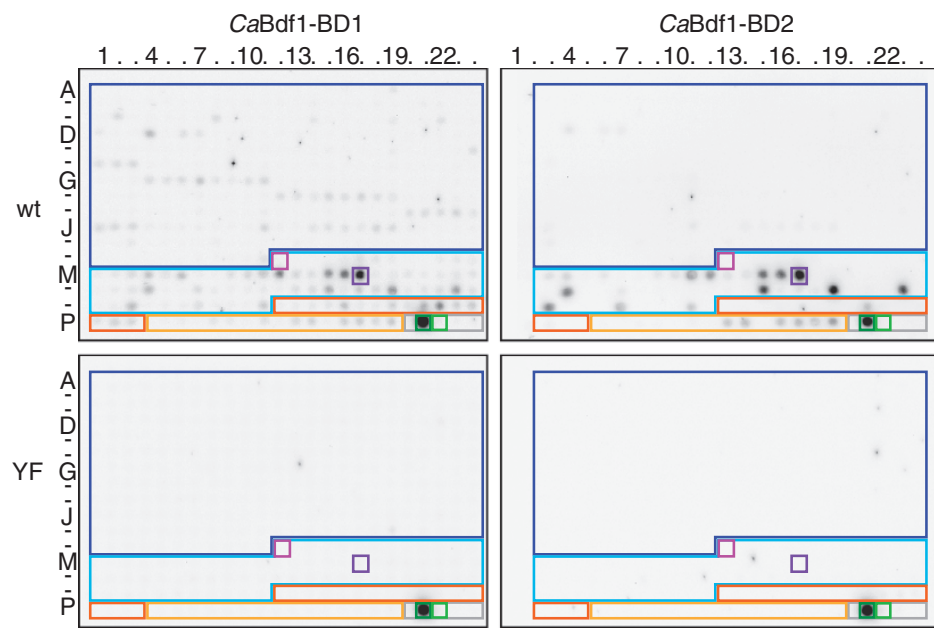

Peptides:
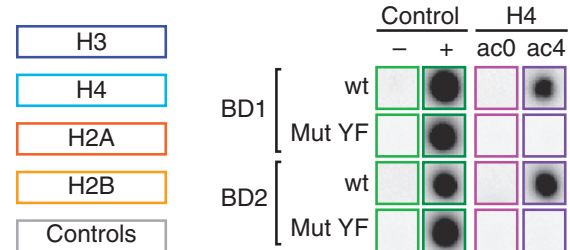

b

$\%$ identity within BD1

\begin{tabular}{|c|c|c|c|c|c|c|c|c|}
\hline & \multicolumn{4}{|c|}{ human } & $\begin{array}{l}\text { S.cer } \\
\text { Bdf1 }\end{array}$ & $\begin{array}{l}\text { C.alb } \\
\text { Bdf1 }\end{array}$ & $\begin{array}{l}\text { C. gla } \\
\text { Bdf1 }\end{array}$ \\
\hline & Brd2 & -- & 87 & 79 & $\overline{74}$ & 35 & 31 & 35 \\
\hline స్ है & Brd3 & 82 & -- & 81 & 74 & 32 & 31 & 33 \\
\hline & Brd4 & 81 & 81 & -- & 73 & 35 & 32 & 34 \\
\hline & Brdt & 72 & 71 & 74 & -- & 33 & 31 & 31 \\
\hline S.cer & Bdf1 & 39 & 38 & 40 & 41 & -- & 66 & 84 \\
\hline C.alb & Bdf1 & 44 & 42 & 44 & 46 & 58 & -- & 61 \\
\hline C.gla & Bdf1 & 40 & 38 & 37 & 38 & 71 & 57 & -- \\
\hline
\end{tabular}

C

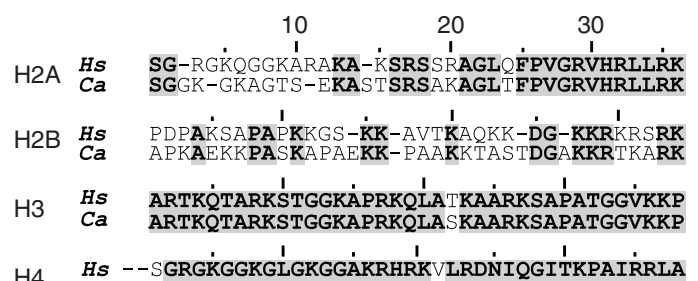

H4 Hs --SGRGKGGKGL'GKGGAंKRHRKVLRDNंIQGITTKPAIRRLA Ca SGTGRGKGGKGLGKGGAKRHRK I LRDNIOGITKPAIRRLA

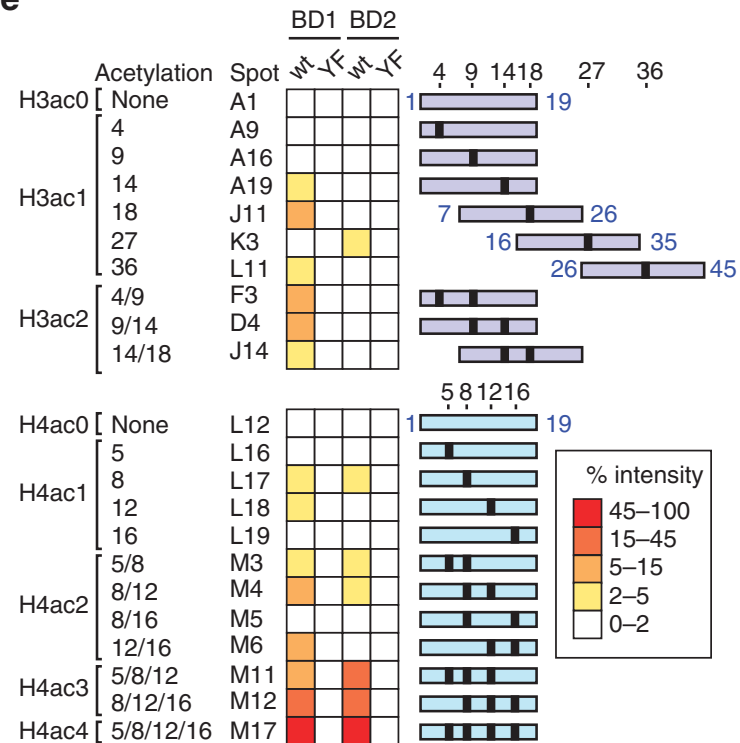

f

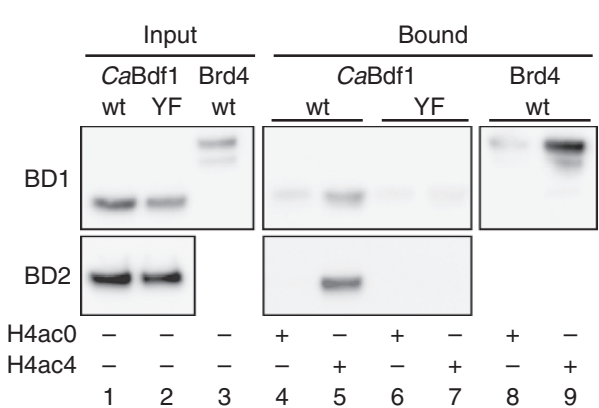

Figure 1 | CaBdf1 BDs bind multi-acetylated histone tails. (a) Phylogeny and domain organization of fungal Bdf1 and human BET proteins. The neighbourjoining tree based on an alignment of BET protein sequences was generated using programs ClustalX and NJPlot. (b) Sequence identity among human and fungal BET BDs. (c) Human ( $\mathrm{Hs}$ ) and C. albicans ( $\mathrm{Ca}$ ) histone tail sequences. (d) Binding of WT and mutant (YF) CaBdf1 BDs to a histone peptide microarray. The array comprised control peptides (grey outline) or $\mathrm{N}$-terminal peptides from histones H3, $\mathrm{H} 4, \mathrm{H} 2 \mathrm{~A}$ and $\mathrm{H} 2 \mathrm{~B}$ (blue, cyan, dark orange and light orange outlines, respectively). Signals for background and positive-binding controls (boxed in light and dark green) or for $\mathrm{H} 4 \mathrm{acO}$ and $\mathrm{H} 4 \mathrm{ac} 4$ peptides (boxed in magenta and purple) are shown magnified below the array. Microarray data are summarized in Supplementary Data 1. (e) Binding intensities for $\mathrm{H} 3$ and $\mathrm{H} 4$ peptides. Peptides are shown as blue (H3) and cyan (H4) rectangles, with acetylation marks indicated by black boxes. (f) Pull-down assay. Immobilized $\mathrm{H} 4 \mathrm{acO}$ and $\mathrm{H} 4 \mathrm{ac} 4$ peptides were incubated with GST-tagged $\mathrm{CaBdf1} \mathrm{BDs}$ or with the corresponding YF mutants. After washing, bound proteins were eluted and visualized by western blotting with an anti-GST antibody. The full blots are shown in Supplementary Fig. 13a. 
a
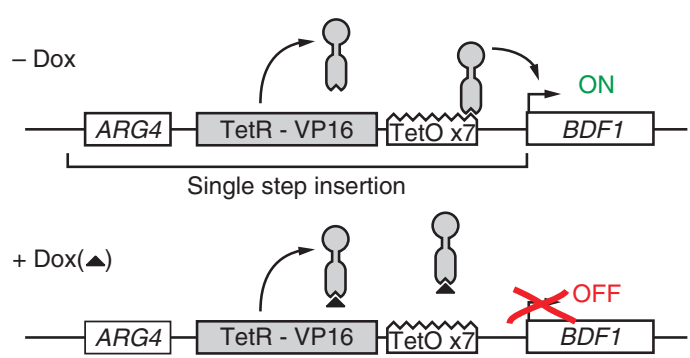

C

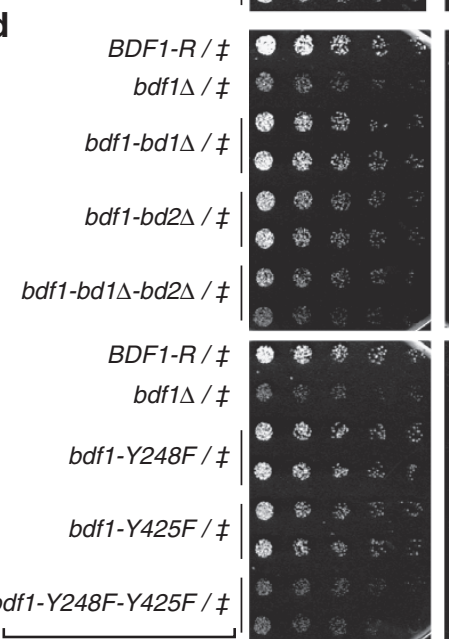

$\neq=p T e t O-B D F 1$

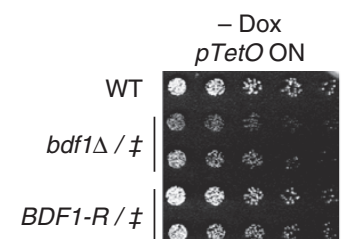

+ Dox

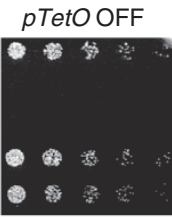

- 1
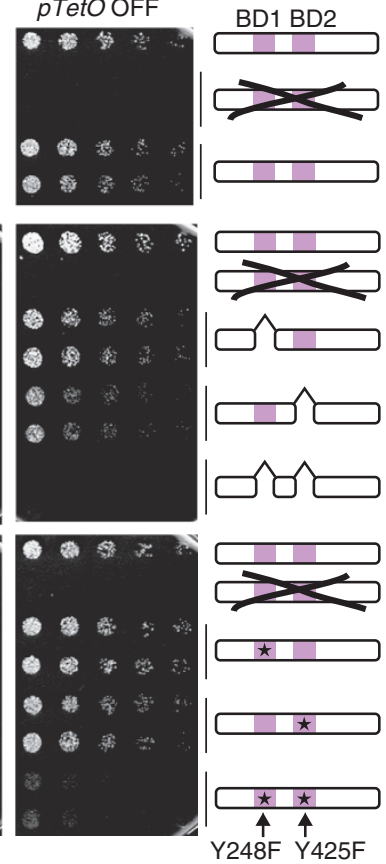

b

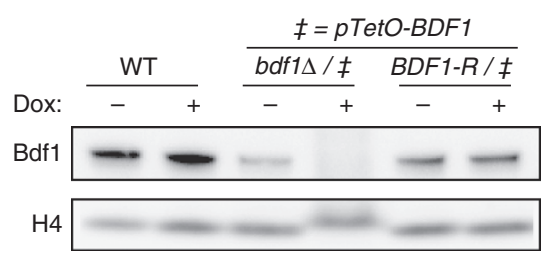

e

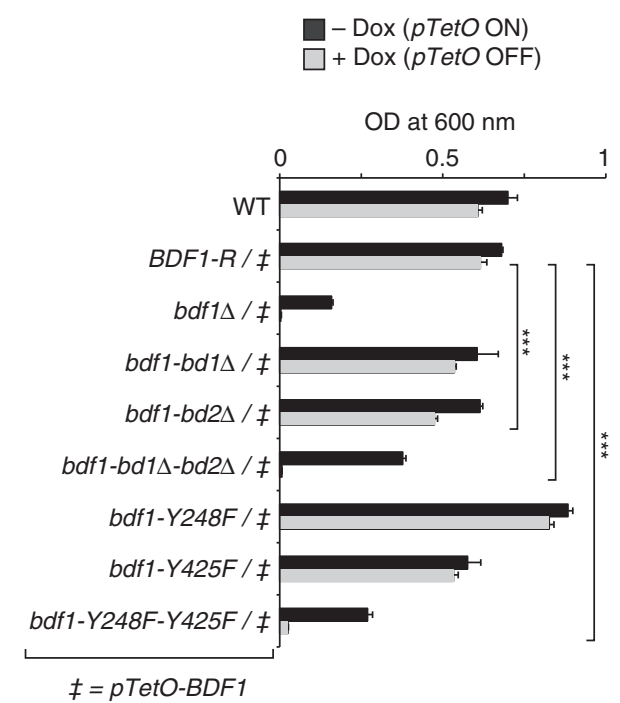

g

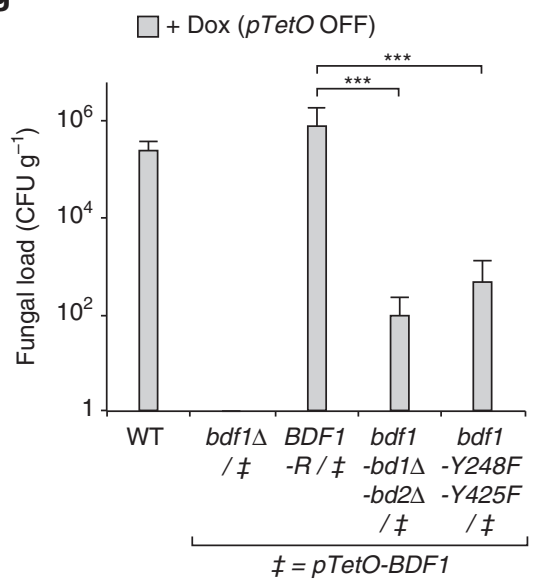

Figure 2 | Bdf1 BDs are essential for C. albicans viability and virulence. (a) Tet-OFF construct used in this study. Dox inhibits the binding of TetR-VP16 to the TetO, preventing BDF1 transcription. (b) Bdf1 protein expression in different strains. The full blots are shown in Supplementary Fig. 13b. (c,d) Colony formation assays showing the effect of (c) Bdf1 repression and (d) Bdf1 BD inactivation on growth. This experiment was repeated three times with similar results. (e) Growth assays in liquid media. An equal fungal load was seeded for each strain and growth monitored by optical density at $600 \mathrm{~nm}$. Mean and s.d. values are shown from three independent experiments. ${ }^{\star \star \star} P \leq 0.01$. $P$ values were determined in a two-sided Welch $t$-test. (f) Kidney fungal load of mice injected with strains shown in (c) measured 7 days post infection, showing that Bdf1 is required for virulence. Data shown are mean and s.d. values $(n=6) .{ }^{\star} P \leq 0.07 ;{ }^{\star \star} P \leq 0.05$. $P$ values were determined using a two-sided Wilcoxon rank sum test with continuity correction. (g) Kidney fungal load of mice infected with strains shown in (d), showing the loss of virulence on BD inactivation. Data shown are mean and s.d. values $(n=6)$. ${ }^{\star \star \star} P \leq 0.01 ; P$ values were determined using a two-sided Wilcoxon rank sum test with continuity correction. 
WT allele is expressed from the Dox-repressible promoter. Strains in which both $\mathrm{BDs}$ were inactivated grew as poorly as the conditional deletion mutant, whereas strains in which only BD1 or $\mathrm{BD} 2$ was inactivated displayed milder growth defects, with $\mathrm{BD} 2$ inactivation yielding the more pronounced defect (Fig. 2d). Additional assays evaluating stress resistance or cell wall integrity did not reveal any significant phenotype. Growth rates in liquid media recapitulated the phenotypes observed in the colony formation assay (Fig. 2e). Analogous results were obtained when $B D F 1$ expression was repressed using the methionine-regulatable promoter (Supplementary Fig. 2). Thus, C. albicans viability in vitro requires the presence of at least one functional $\mathrm{BD}$ within Bdf1.

Bdf1 BDs are required for virulence in a mouse model. Using our Tet-OFF system for Dox-repressible Bdf1 expression, we verified the role of Bdf1 in a murine model of invasive candidiasis. Initially, mice were injected with either WT C. albicans, the conditional $B D F 1$ deletion strain $(b d f 1 \Delta / p T e t O-B D F 1)$ or the rescue strain (BDF1-R/pTetO-BDF1). Mice injected with the WT or rescue strains exhibited a high fungal load $\left(>110,000 \mathrm{CFU} \mathrm{g}^{-1}\right)$ in the kidney 7 days post infection (Fig. $2 \mathrm{f}$ ). The fungal load observed with strain $b d f 1 \Delta / p T e t O-B D F 1$ was reduced by 30 -fold $\left(\sim 3,500 \mathrm{CFU} \mathrm{g}^{-1}\right)$ in the absence of Dox and was abolished in its presence, mirroring the growth phenotypes observed in vitro (Fig. 2c). Subsequently, we evaluated the virulence of strains in which both Bdf1 BDs were deleted or inactivated by the YF point mutations (Fig. 2g). These strains exhibited markedly reduced fungal loads (by $\sim 8,800$ - and 1,600-fold, respectively), consistent with the in vitro growth rates associated with these mutations (Fig. 2d,e and Supplementary Fig. 2). Hence, Bdf1 BD functionality appears critical for the virulence of C. albicans in vivo.

CaBdf1 BDs are resistant to human BET inhibitors. For Bdf1 inhibition to be a feasible antifungal therapeutic strategy, smallmolecule inhibitors need to discriminate fungal from human BET $\mathrm{BDs}$. To probe the similarity between the ligand-binding pockets of $C$. albicans and human BET BDs, we asked whether human BET inhibitors (BETi) could inhibit CaBdf1 BDs. We used a homogeneous time-resolved fluorescence (HTRF) assay to evaluate the ability of four chemically diverse BETi compounds (JQ1, PFI-1, IBET-151 and bromosporine) to inhibit BD binding to an H4ac4 peptide (Fig. 3a). The four compounds inhibited human Brd4 BD1 and BD2 with median inhibitory concentrations $\left(\mathrm{IC}_{50}\right.$ values) in the low $\mathrm{nM}$ range $(3-95 \mathrm{nM})$, consistent with previous reports ${ }^{16,35,36}$. In contrast, the CaBdf1 BDs were relatively insensitive to these inhibitors, with $\mathrm{IC}_{50}$ values between 0.3 and $>10 \mu \mathrm{M}$, or approximately a two- to threefold logreduction in sensitivity (Fig. 3b). These results were confirmed for JQ1 by isothermal titration calorimetry (ITC): whereas JQ1 bound Brd4 BD1 tightly ( $K_{\mathrm{d}}$ of $\left.62 \mathrm{nM}\right)$, no binding was detected for CaBdf1 BD1 or BD2 (Fig. 3c and Supplementary Table 1). None of the BETi compounds significantly inhibited growth of C. albicans in vitro at $10 \mu \mathrm{M}$ concentration (Fig. 3d). This finding is consistent with the poor $\mathrm{IC}_{50}$ values observed for JQ1 and PFI1 towards CaBdf1 BDs. It also indicates that IBET-151 and bromosporine, which display (sub)micromolar $\mathrm{IC}_{50}$ values, have poor cellular potency, possibly due to mechanisms known to reduce drug potency in C. albicans, including cell wall and plasma membrane permeability barriers ${ }^{37,38}$ and the activity of efflux pumps leading to rapid drug extrusion ${ }^{39,40}$. In summary, $\mathrm{CaBdf1}$ and human BET BDs have distinct BETi-binding activity, supporting the feasibility of targeting CaBdf1 BDs with highly selective small-molecule inhibitors.
BETi resistance by $\mathrm{CaBdf1}$ is due to smaller pocket residues. To identify features differentiating the ligand-binding pockets of human and fungal BET BDs, we determined crystal structures for $\mathrm{CaBdf1} \mathrm{BD} 1$ and BD2 (Supplementary Table 2). These structures exhibit the canonical BD fold (comprising helices $\mathrm{Z}, \mathrm{A}, \mathrm{B}$ and $\mathrm{C}$, with the $\mathrm{ZA}$ and $\mathrm{BC}$ loops defining the ligand-binding pocket; Fig. 4a) and closely resemble those of their human BET counterparts (mean pairwise root-mean-square deviations (RMSDs) $\leq 1.5 \AA ̊$; Supplementary Table 3). The ligand-binding loops are structurally well conserved, including nearly all water molecules implicated in ligand recognition (Supplementary Fig. 3), although the overall binding surfaces are less negatively charged in $\mathrm{CaBdf1}$ (Fig. 4b). Superimposing the CaBdf1 BD structures onto that of JQ1-bound Brd4 BD1 reveals no major steric clashes between JQ1 and the fungal BDs. However, whereas JQ1 fits snugly into the Brd4-binding pocket, the fit with the CaBdf1-binding pockets is poor. In Brd4 the fused ring system of JQ1 is sandwiched between the 'WPF' shelf (Trp81, Pro82 and Phe83) and Leu residues 92 and 94 , with the thiophene ring positioned within the 'ZA channel' defined by Trp81 and Leu92 (ref. 41). Replacement of Trp81 by a Val or Phe residue in CaBdf1 BD1 and BD2, respectively, widens the ZA channel, resulting in a poorer fit (black diamonds in Fig. 4c). Replacement of Brd4 residue Leu94 by a Val or Ile residue further contributes to the poor fit. In addition, the p-chlorophenyl group of JQ1 occupies a groove in Brd4, which is absent (BD1) or shallower (BD2) in CaBdf1 (asterisks in Fig. 4c). A similar lack of complementarity between the ligand and binding pocket explains the insensitivity of $\mathrm{CaBdf1}$ BDs towards PFI-1, IBET-151 and bromosporine.

More generally, recognition of the four BETi compounds by human BET BDs involves 16 residues, including the eight signature residues previously used to classify human BD-binding $\operatorname{sites}^{42}$ (Fig. 5). Five of these residues in BD1 and four in BD2 are invariant across human BET proteins but diverge in CaBdf1 (magenta arrows in Fig. 5), including the WPF-shelf Trp residue and the Leu residue opposite this shelf, at signature positions 1 and 3 , respectively. Remarkably, nearly all of these residues have shorter side chains in CaBdf1 than in the human BET proteins, implying that selective CaBdf1 inhibition could conceivably be achieved via small molecules that clash sterically with the human, but not fungal, side chains. Interestingly, other pathogenic fungal Bdf1 sequences also diverge from human BET proteins at these residue positions, suggesting that selective chemical inhibition might also be feasible for these targets.

CaBdf1 BDs can be selectively targeted by small molecules. We next set out to identify small-molecule inhibitors that would target C. albicans Bdf1 BDs without inhibiting human BET BDs. We used our HTRF inhibition assay to screen a library of $\sim 80,000$ chemically diverse compounds, resulting in several hundred hits for each BD (Fig. 6a). Dose-response curves measured for $\mathrm{CaBdf} 1$ and Brd4 BDs identified 125 and 44 compounds selective for the fungal $\mathrm{BD} 1$ and $\mathrm{BD} 2$, respectively. In particular, several compounds possessing a dibenzothiazepinone scaffold exhibited low-micromolar $\mathrm{IC}_{50}$ values towards $\mathrm{CaBdf1}$ BD1 and no or only weak inhibition of $\mathrm{Brd} 4 \mathrm{BD} 1$ at the highest concentration $(20 \mu \mathrm{M})$ tested. One such inhibitor was compound 1 (Fig. 6b). ITC measurements showed that 1 binds CaBdf1 BD1 with a $K_{\mathrm{d}}$ of $5 \mu \mathrm{M}$, consistent with the $\mathrm{IC}_{50}$ value $(4.3 \mu \mathrm{M})$ determined by HTRF, whereas no or only modest binding and inhibition were detected for Brd4 BD1 and CaBdf1 BD2 (Fig. 6c,d and Supplementary Table 1). Furthermore, a BROMOscan screen from DiscoverX revealed that compound $\mathbf{1}$ showed no significant activity against 32 representative human (BET and non-BET) BDs (Supplementary Fig. 4); indeed, an ITC experiment 
a

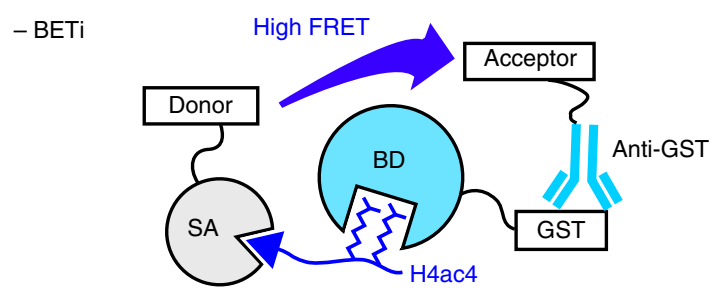

$+\mathrm{BETi}$

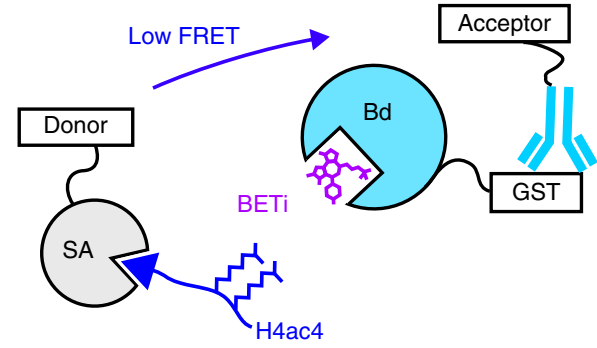

C
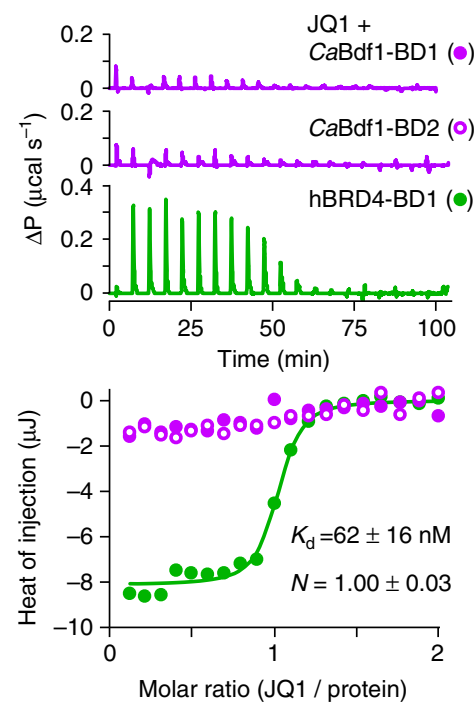

b

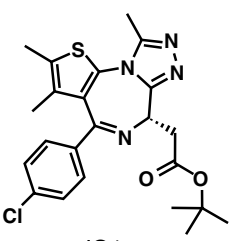

JQ1

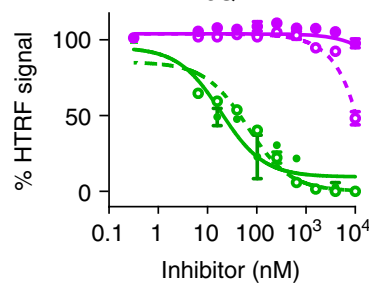

$\mathrm{IC}_{50}$
values
(nM) $\left[\begin{array}{cccr}\text { CaBFD1 } & \text { BD1 } & - & >10,000 \\ " & \text { BD2 } & -\bullet- & \sim 10,000 \\ \text { hBRD4 } & \text { BD1 } & \bullet & 18.7 \pm 11.2 \\ " & \text { BD2 } & -\bullet- & 64.9 \pm 8.1\end{array}\right.$<smiles></smiles>

PFI-1

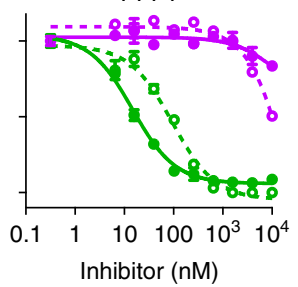

$\rightarrow>10,000$

- $\bullet-\sim 10,000$

$\rightarrow-14.7 \pm 3.3$

$=0-94.8 \pm 2.8$<smiles></smiles>

IBET-151

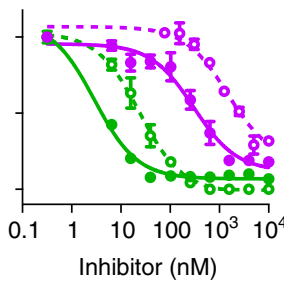

$\rightarrow \quad 303 \pm 120$

- o- $1470 \pm 90$

$\because \quad 2.9 \pm 0.1$

- o- $24.1 \pm 1.1$

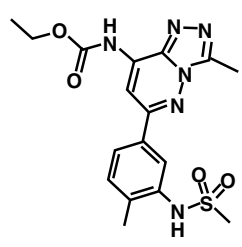

Bromosporine

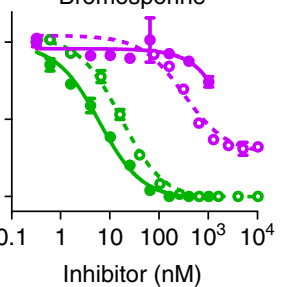

$\rightarrow \quad>10,000$

- o- $313 \pm 2$

$\rightarrow \quad 6.0 \pm 0.7$

- o- $16.5 \pm 1.5$

d

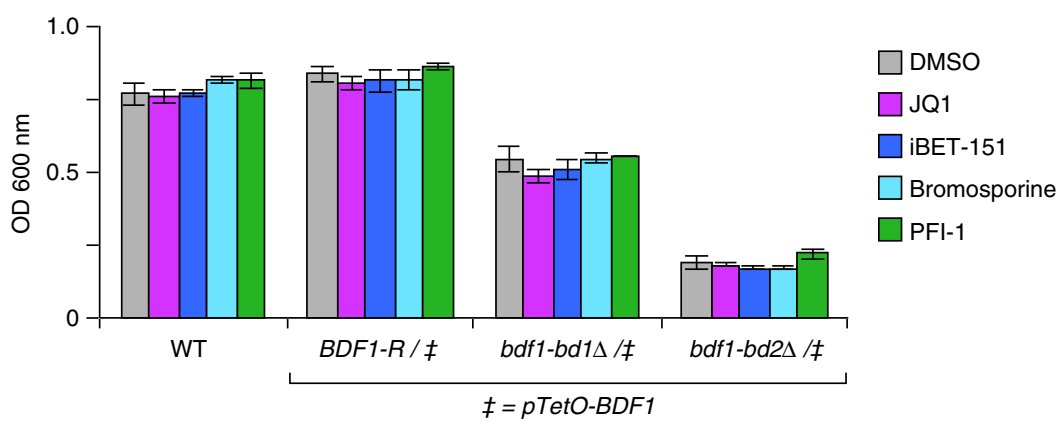

Figure 3 | CaBdf1 BDs are resistant to BETi. (a) HTRF assay. A biotinylated tetra-acetylated histone $\mathrm{H} 4$ peptide is bound to streptavidin beads coupled to the donor fluorophore. A GST-tagged BD is bound by an anti-GST antibody coupled to the acceptor fluorophor. Peptide binding by the BD results in FRET. The addition of a BETi reduces FRET. (b) HTRF assays performed on BDs from CaBdf1 and human Brd4 in the presence of the indicated BETi. Inhibition curves are shown as closed (BD1) and open (BD2) circles in green (Brd4) and magenta (Bdf1). IC $C_{50}$ values are listed below each graph. Data represent the mean and s.d. values from three independent experiments. (c) Representative ITC experiments measuring the binding of JQ1 to CaBDF1 BDs (magenta) and to human Brd4 BD1 (green). The values indicated for $K_{d}$ and $N$ represent the mean and s.d. from three independent experiments. See also Supplementary Table 1. (d) BET inhibitors do not affect C. albicans growth, even when Bdf1 BD1 or BD2 is deleted. Inhibitors were tested at $10 \mu \mathrm{M}$ concentration. Experiments were performed in the presence of doxycyline to repress expression from the pTetO-BDF1 allele. Data represent the mean and s.d. values from three independent experiments. 
a
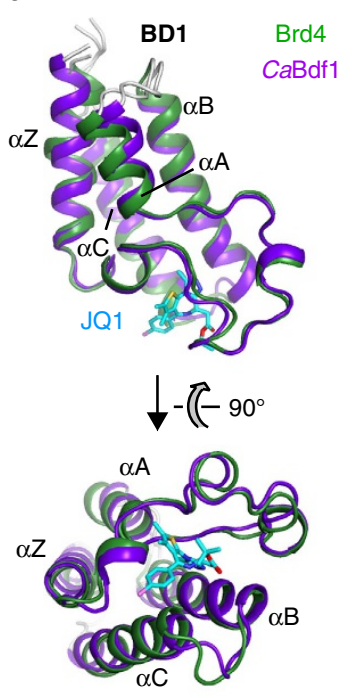
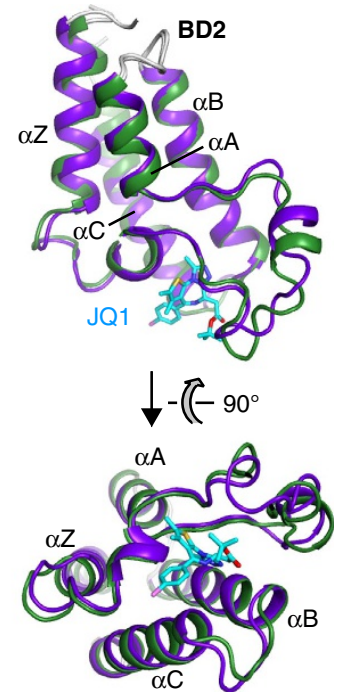

b

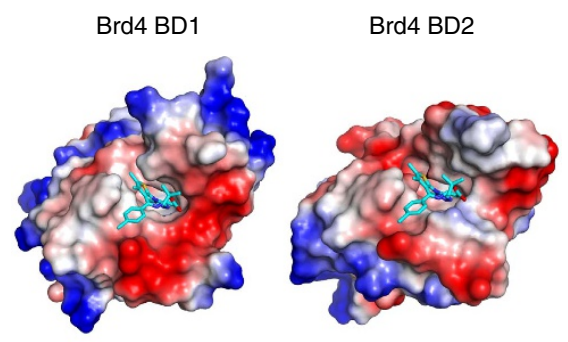

CaBdf1 BD1

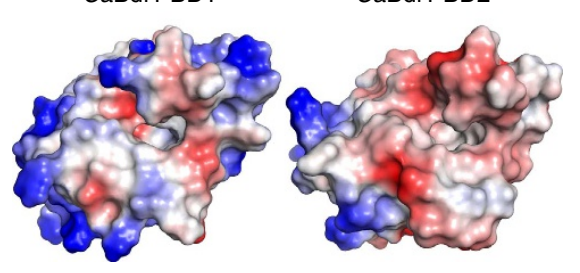

C
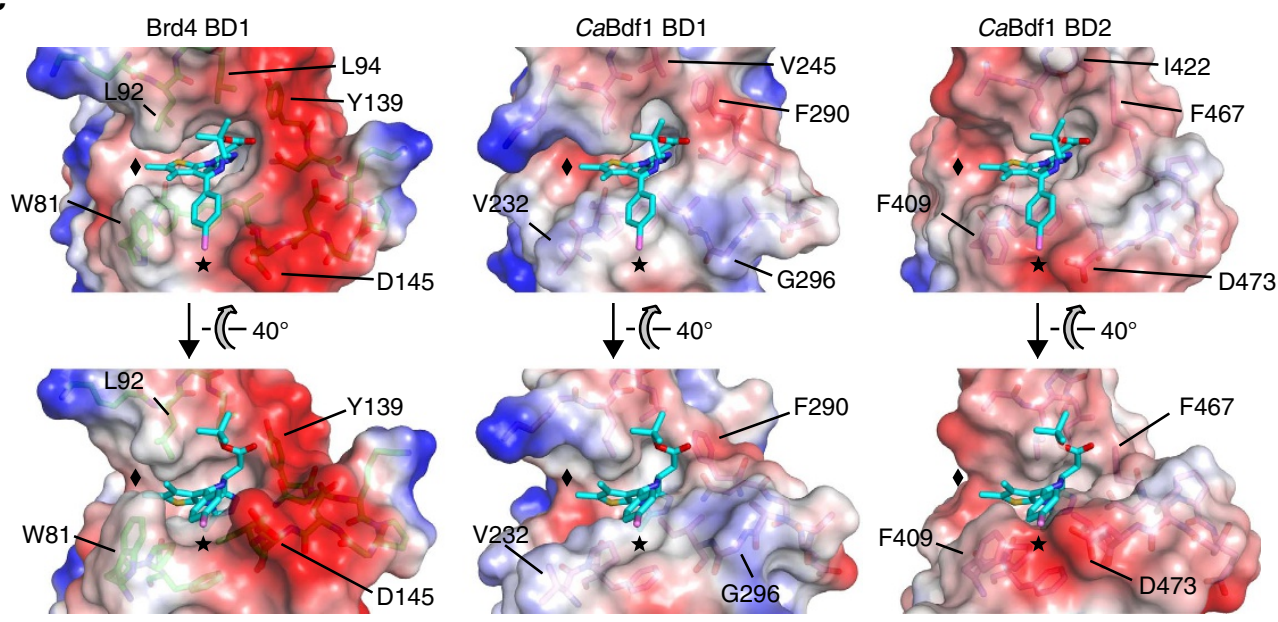

Figure 4 | Structural basis of BETi resistance by CaBdf1 BD1 and BD2. (a) Alignment of BD1 (left) and BD2 (right) structures from human Brd4 (green; PDB codes $3 \mathrm{MXF}$ and 2OUO) and CaBdf1 (magenta). For clarity, the 18 and $20 \mathrm{~N}$-terminal residues preceding helix $\alpha \mathrm{Z}$ are omitted from the Brd4 BD1 and CaBdf1 BD1 structures, respectively. Brd4-bound JQ1 is shown in cyan. (b) Plots of electrostatic surface potential of BD1 (left) and BD2 (right) structures from human Brd4 and CaBdf1. Regions of negative and positive potential are shown in red and blue, respectively. JQ1 bound to Brd4 is shown in cyan. The binding surfaces of the fungal BDs are less negatively charged compared to human BET BDs, suggesting differences in the binding partners of these domains. (c) Comparison of the ligand-binding pockets of CaBdf1 BD1 and BD2 with that of Brd4 BD1. JQ1 was superimposed on the CaBdf1 BDs via a structural alignment with Brd4 BD1. The diamond and asterisk indicate the ZA channel and surface groove which in Brd4 BD1 interact with the thieno and p-chlorophenyl groups of JQ1, respectively, and the corresponding positions in the CaBdf1 BDs. The view in the upper panels is that of (b) rotated $50^{\circ}$ counter-clockwise.

confirmed that the two human BDs (SMARCA2 and SMARCA4) with the strongest BROMOscan response did not detectably bind compound 1 (Supplementary Fig. 5a). Compound 1 also exhibited low cytotoxicity towards HeLa and IMR90 (primary fibroblast) cells as measured in an MTT colorimetric assay (EC $\mathrm{E}_{50} \geq 100 \mu \mathrm{M}$; Supplementary Fig. 6).

To understand the molecular basis of selectivity we solved the crystal structure of $\mathrm{CaBdf1} \mathrm{BD} 1$ bound to $\mathbf{1}$. Compound 1 mimics the acetyllysine ligand by interacting with the conserved Tyr248 and Asn291 residues, forming direct and water-mediated hydrogen bonds via the thiazepinone carbonyl group (Fig. 6e-g). The dibenzothiazepinone ring system engages hydrophobic residues from the Val-Pro-Phe (VPF) shelf (Pro233), helix B (Phe290) and the ZZ' loop (Val238, Leu243 and Val245), while the cyclopropyl group interacts with the VPF shelf (Pro233 and Phe234), helix B (Cys287) and the gatekeeper residue on helix C (Ile297). In contrast, the oxadiazole and pyrazine groups point away from the pocket, adopting different orientations in the four complexes present in the crystal's asymmetric unit (Supplementary Fig. 7). Superimposing the ligand-bound structure with that of Brd4 BD1 results in a steric overlap between the larger Brd4 side chains at signature positions 1 and 3 (Trp81 and Leu94) and the oxadiazole and distal benzene groups of compound 1, explaining why $\mathbf{1}$ fails to inhibit the human $\mathrm{BD}$ (Fig. 6f,h). Steric hindrance from the corresponding residues in CaBdf1 BD2 (Phe409 and Ile422) also account for this domain's insensitivity to compound $\mathbf{1}$.

Similarly, we identified an imidazopyridine compound 2 (Fig. 7a), which inhibits CaBdf1 BD2 with high selectivity: HTRF and ITC assays yielded $\mathrm{IC}_{50}$ and $K_{\mathrm{d}}$ values of 1.1 and $2.1 \mu \mathrm{M}$, respectively, whereas human $\mathrm{Brd} 4 \mathrm{BD} 2$ and $\mathrm{CaBdf1} \mathrm{BD} 1$ were only weakly inhibited $\left(\mathrm{IC}_{50} \geq 40 \mu \mathrm{M}\right)$ (Fig. $7 \mathrm{~b}, \mathrm{c}$ and Supplementary Table 1). Moreover, no significant inhibition of any human BDs was observed for compound 2 by BROMOscan 


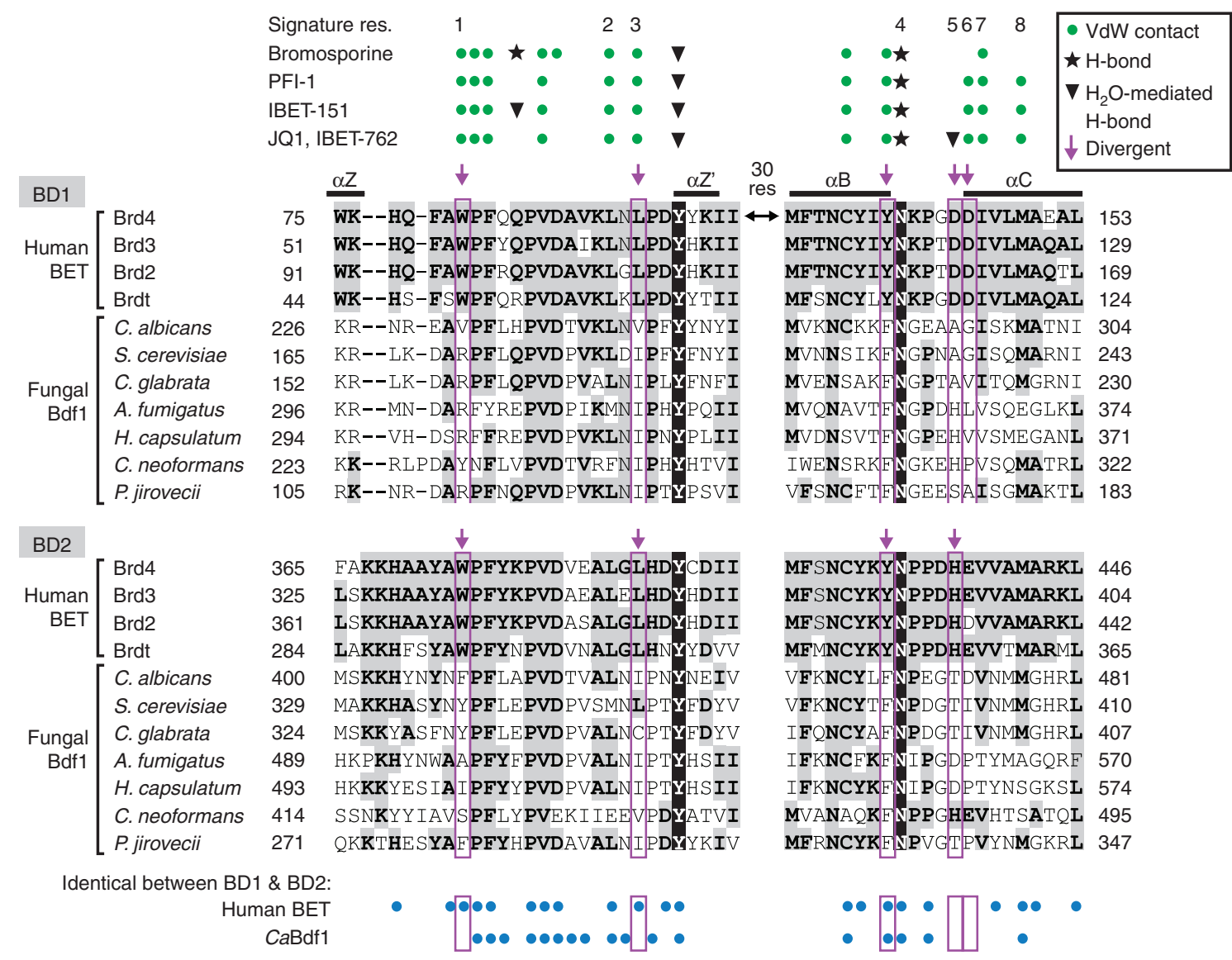

Figure 5 | Structure-based sequence alignment of human and fungal BET proteins showing the ligand-binding region. Residues highlighted in grey are conserved in at least three human BET sequences within each BD. The conserved Tyr and Asn residues involved in water-mediated and direct hydrogen bonds to the ligand are shown in inverse font. Human BET BD residues interacting with BETi compounds through direct or water-mediated hydrogen bonds or through van der Waals contacts are indicated by asterisks, arrowheads and green circles, respectively. Interactions are taken from the crystal structures of Brd4 BD1 bound to JQ1, IBET-762 or PFI-1 (PDB codes 3MXF, 3P5O and 4E96), from that of Brd2 BD1 bound to IBET-151 (PDB 4ALG), and from a structural alignment of Brd4 BD1 with human BRPF1 BD bound to bromosporine (PDB 4C7N). The signature residues used to classify human BD-binding sites are numbered 1-8 (ref. 42). Magenta arrows and boxes indicate BETi-contacting residues which are conserved in human BET proteins but differ in $\mathrm{CaBdf1}$ and other fungal sequences. Blue dots below the alignment indicate residues which are identical between BD1 and BD2 across human BET proteins or within CaBdf1.

profiling and by an ITC assay on the two most sensitive BDs identified by this screen (SMARCA2 and SMARCA4; Supplementary Figs 4 and 5b); while only mild cytotoxicity was observed towards mammalian cells $\left(\mathrm{EC}_{50} \sim 60 \mu \mathrm{M}\right.$; Supplementary Fig. 6). The crystal structure of CaBdf1 BD2 bound to 2 reveals that the compound forms a hydrogen bond via its amino group with the conserved Asn468 residue, as well as a cation-pi interaction involving the phenolic ring and the partial positive charge on Asn468 (Fig. 7d-f). However, unlike most BET inhibitors, a water-mediated hydrogen bond with Tyr425 is not observed for $\mathbf{2}$ (presumably because the ligand dislodges water molecule 1 , consistent with the dislodgement of this water by a glycerol molecule in the unbound BD structure; Supplementary Fig. 3d). Instead, the compound's imidazolyl and hydroxyl groups form water-mediated hydrogen bonds with backbone atoms of the Phe-Pro-Phe (FPF) shelf (Pro410) and helix B (Val460). Replacement of the hydroxyl group in $\mathbf{2}$ by a hydrogen (2a) or fluorine (2b) atom severely reduces inhibitory activity, underscoring the importance of this group for high-affinity binding (Supplementary Fig. 8). The methylpyridinyl and phenolic groups are sandwiched between the FPF shelf and gatekeeper residue Val474 on one side, and residues Val415 and Leu420 on the other, while the tolyl group additionally contacts residues Ile 422 and Phe467 (Fig. 7d-f). A structural alignment with Brd4 BD2 shows that larger Brd4 side chains at four of these contact positions, including signature positions 1,3 and $5(\operatorname{Trp} 374$, Leu387 and His437) and residue Tyr432 (Fig. 7e,g), reduces the volume available for compound $\mathbf{2}$, explaining the inability of $\mathbf{2}$ to inhibit the human BD. In contrast, the poor affinity for $\mathrm{CaBdf1}$ BD1

is due to smaller side chains at signature positions 1 and 3 (Val232 and Val245), resulting in poor complementarity between the binding pocket and ligand. The above results establish proofof-principle that CaBdf1 BDs can be selectively targeted by small-molecule inhibitors in vitro without compromising the function of human $\mathrm{BD}$-containing proteins.

A Bdf1 BD1 inhibitor phenocopies genetic inactivation of BD1. We next assessed the antifungal activity of compounds identified in our screen by evaluating their effect on C. albicans growth in liquid media. Not surprisingly, none of the compounds significantly inhibited growth of the WT strain, consistent with the fact that inactivating a single CaBdf1 $\mathrm{BD}$ only weakly affects growth (Fig. 2d,e) and that none of the compounds tested can individually inhibit both $\mathrm{CaBdf1}$ BDs. Attempts to simultaneously inhibit both BDs by testing binary combinations of compounds also did not result in significant growth inhibition. 
a

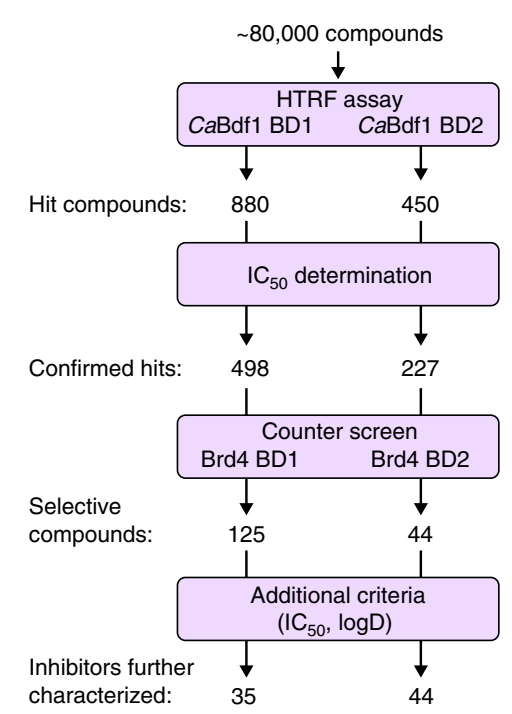

b<smiles>O=C1c2ccccc2Sc2cc(-c3noc(-c4cnccn4)n3)ccc2N1C1CC1</smiles>

C

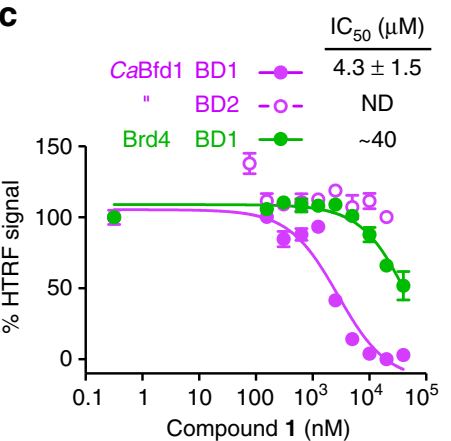

d
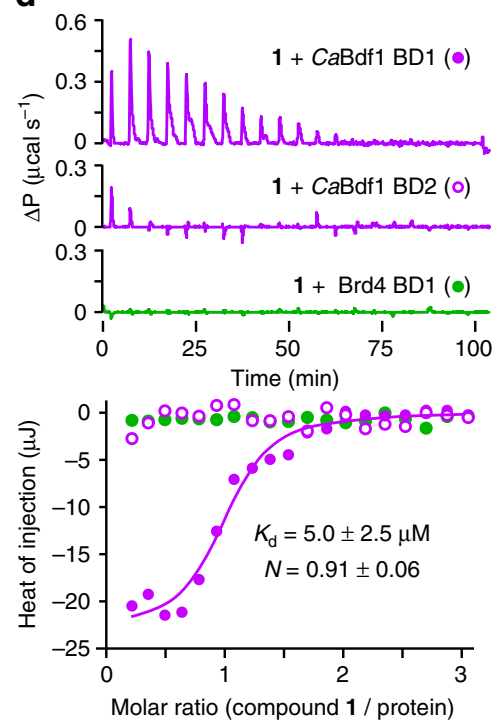

e

f
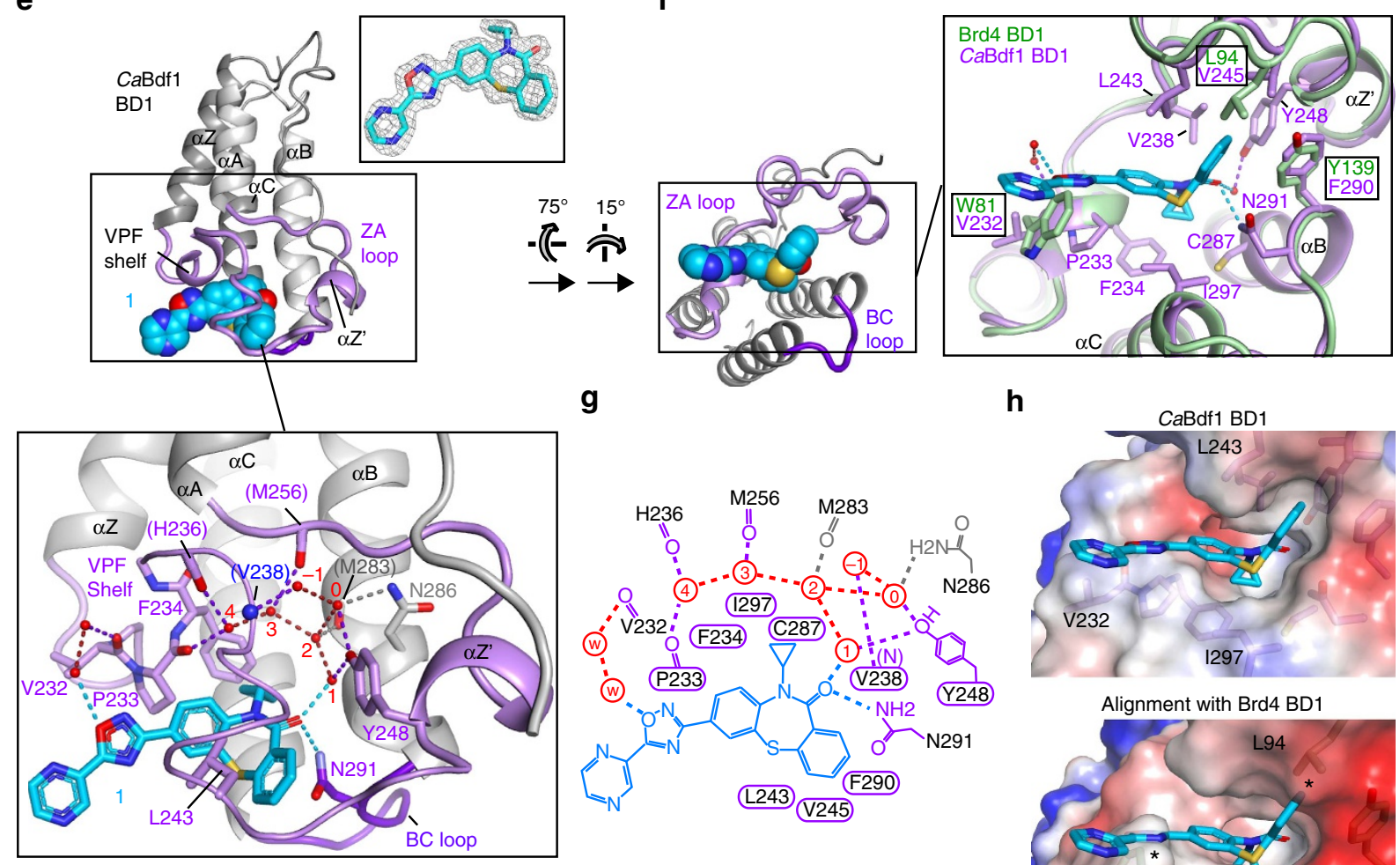

h

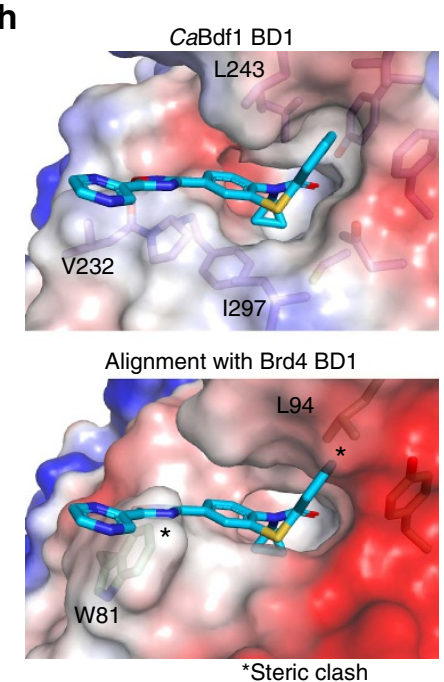

Figure 6 | Identification of a selective CaBdf1 BD1 inhibitor. (a) Screening strategy to identify selective CaBdf1 BD1 and BD2 inhibitors. Following the counter-screen step, most compounds yielded at least a 20 -fold difference in $I_{50}$ value relative to the human $B D$, although a few exhibited only a three- to fourfold difference. (b) Chemical structure of 1. (c) HTRF assays showing selective inhibition of CaBdf1 BD1 by 1. (d) Representative ITC experiments showing selective binding of $\mathbf{1}$ to $\mathrm{CaBdf1}$ BD1. The values indicated for $K_{\mathrm{d}}$ and $N$ represent the mean and s.d. from three independent experiments. See also Supplementary Table 1. (e) Crystal structure of CaBdf1 BD1 bound to 1. Upper inset. Simulated-annealing omit $F_{\mathrm{o}}-F_{\mathrm{c}}$ density for $\mathbf{1}$ contoured at $3 \sigma$. Lower inset. Conserved water structure and hydrogen bonding interactions in the binding site. Residues interacting with $\mathbf{1}$ through direct and water-mediated hydrogen bonds (dashed lines) are shown in stick representation. Residues interacting through backbone atoms are labelled in parentheses. Water molecules are numbered as in ref. 70. (f) Alignment of CaBdf1 BD1 (violet) with Brd4 BD1 (green; PDB code 3UVW). Side chains are shown for CaBdf1 residues in contact with $\mathbf{1}$ and for the corresponding Brd4 residues if divergent from CaBdf1. (g) Schematic summary of ligand interactions. Hydrogen bonds are shown as dashed lines. Residues mediating van der Waals contacts with $\mathbf{1}$ are indicated by labels within a cartouche. Water molecules are indicated in red. (h) Surface representation showing the binding pocket of CaBdf1 BD1 bound to compound $\mathbf{1}$ (top) and that of Brd4 BD1 (bottom) superimposed on the fungal complex. Asterisks indicate steric overlap between compound $\mathbf{1}$ and Brd4 BD1 residues. 
a<smiles>CC1=CC=CN2C1NC(c1ccc(O)cc1)C2Nc1ccc(C)cc1</smiles>

b

$$
\begin{aligned}
& \text { CaBDF1 BD1 } \rightarrow \frac{\mathrm{IC}_{50}(\mu \mathrm{M})}{>20}
\end{aligned}
$$

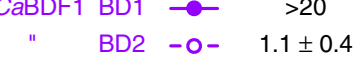

$$
\begin{aligned}
& \text { hBRD4 BD2 } \rightarrow \quad>20
\end{aligned}
$$
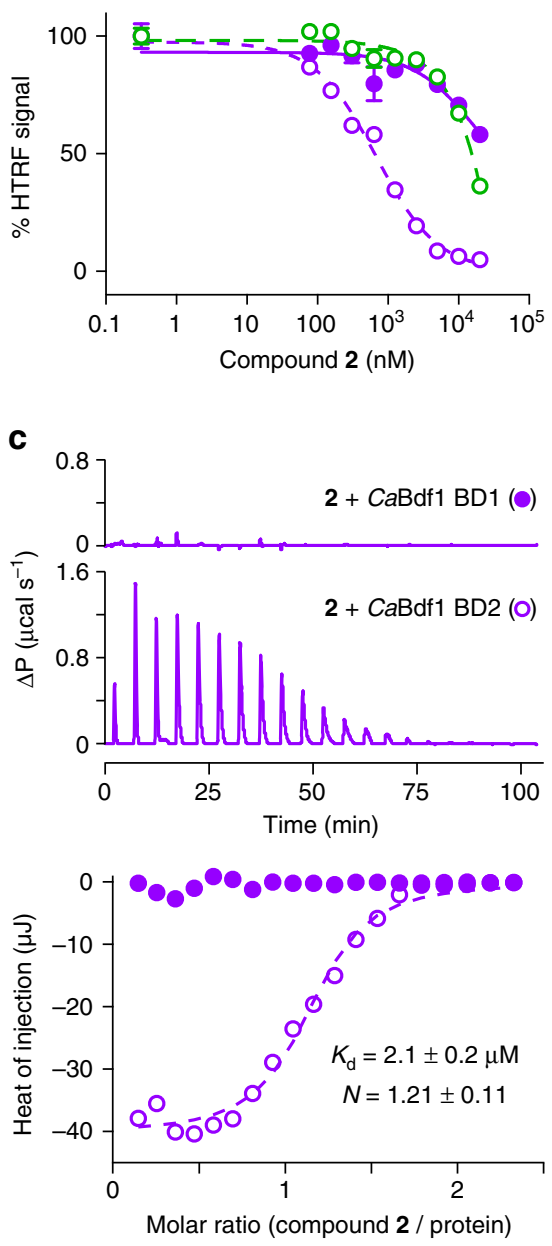

d

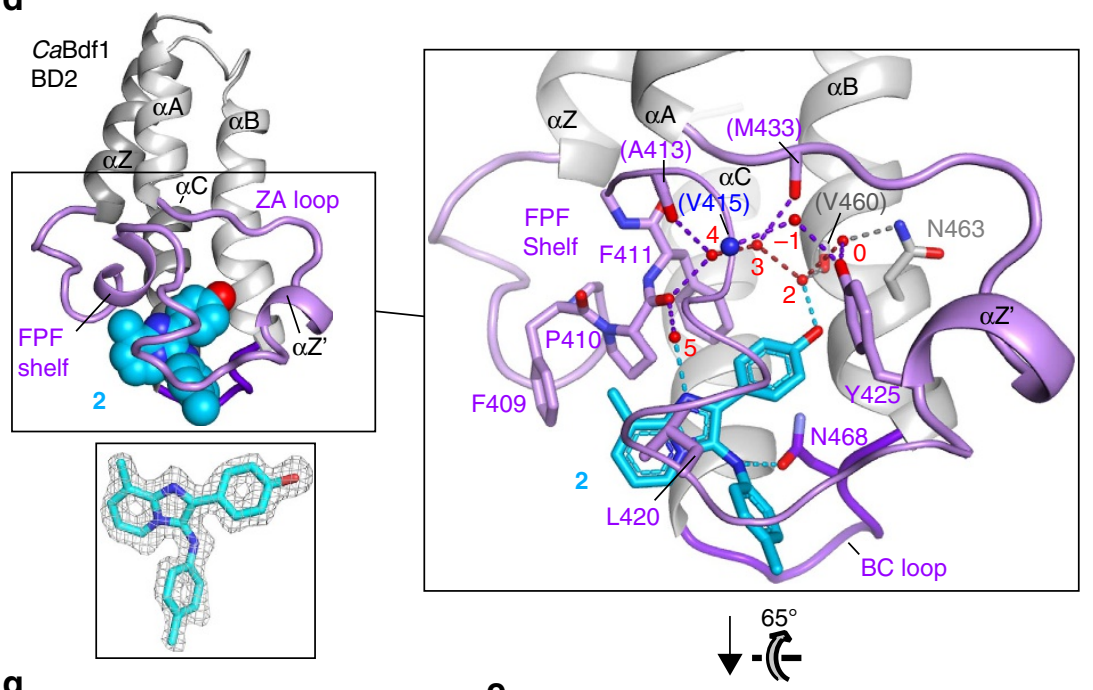

CaBdf1 BD2

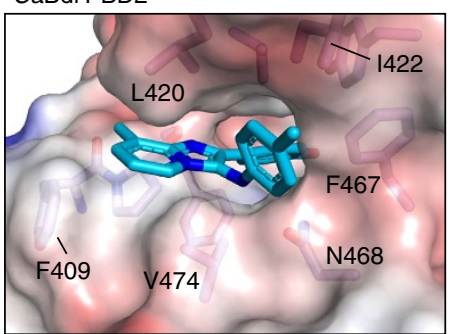

Brd4-BD2

CaBdf1-BD2

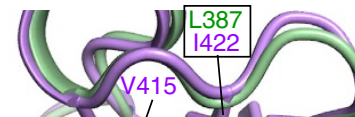

L420

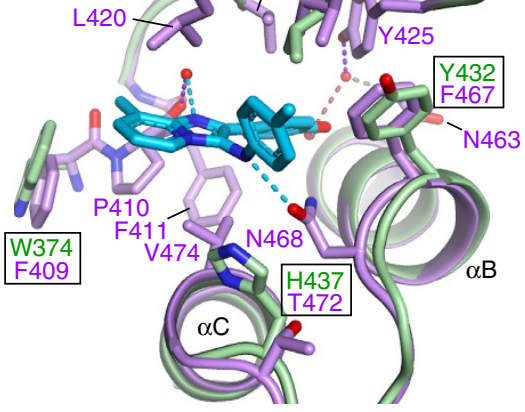

f

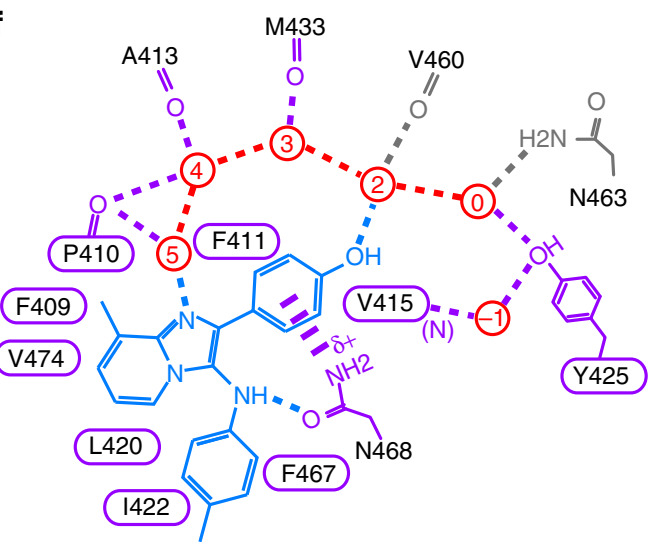

Figure 7 | Identification of a selective CaBdf1 BD2 inhibitor. (a) Chemical structure of compound 2. (b) HTRF assays showing selective inhibition of CaBdf1 BD2 by 2. (c) Binding of $\mathbf{2}$ to CaBdf1 BDs measured by ITC. The values indicated for $K_{\mathrm{d}}$ and $N$ represent the mean and s.d. from three independent experiments. See also Supplementary Table 1. (d) Crystal structure of CaBdf1 BD2 bound to 2. Lower inset. Simulated-annealing omit $F_{\mathrm{o}}-F_{\mathrm{c}}$ density for $\mathbf{2}$ contoured at $3 \sigma$. Right inset. Details of the binding site. Residues interacting with $\mathbf{2}$ through direct and water-mediated hydrogen bonds (dashed lines) are shown in stick representation. Residues interacting through backbone atoms are labelled in parentheses. Water molecules are numbered as in ref. 70. (e) Alignment of CaBdf1 BD2 (violet) with Brd4 BD2 (green; PDB code 4Z93). Side chains are shown for CaBdf1 residues in contact with 2 and for the corresponding Brd4 residues if divergent from CaBdf1. (f) Schematic summary of interactions. Hydrogen bonds are shown as dashed lines. Water molecules are in red. The cation-pi type interaction between the partially charged N468 amino group and the phenol ring is shown as a thick dashed line. Residues mediating van der Waals contacts with $\mathbf{2}$ are indicated by labels within a cartouche. (g) Surface representation showing the binding pocket of CaBdf1 BD2 bound to compound $\mathbf{2}$ (top) and that of Brd4 BD2 (bottom) superimposed on the fungal complex. Asterisks indicate close contacts predicted to sterically inhibit the recognition of $\mathbf{2}$ by Brd4 BD2. Double arrows indicate short distances which may contribute additional steric hindrance.

We therefore tested individual compounds on mutant strains in which either BD1 or BD2 was inactivated. Most inhibitors, including compounds 1 and 2, showed little antifungal activity against these strains. We speculate that the compounds failed to enter the fungal cell because of cellular permeability barriers ${ }^{37,38}$, or were extruded by efflux pumps 39,40 or metabolized before a 
a<smiles>CN1C(=O)c2ccccc2Sc2cc(NC(=O)C3CCCO3)ccc21</smiles>

3

b
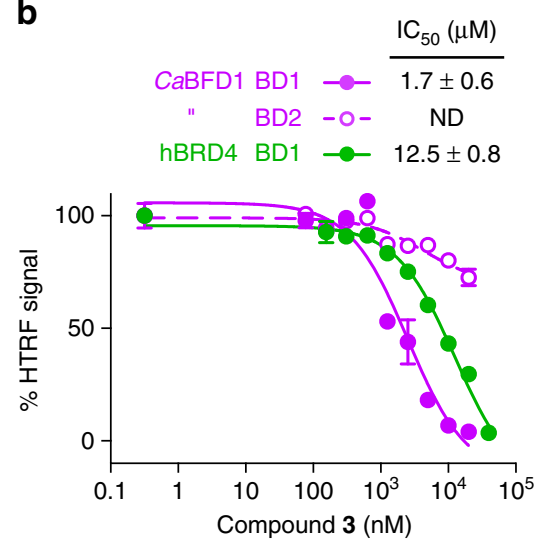

e
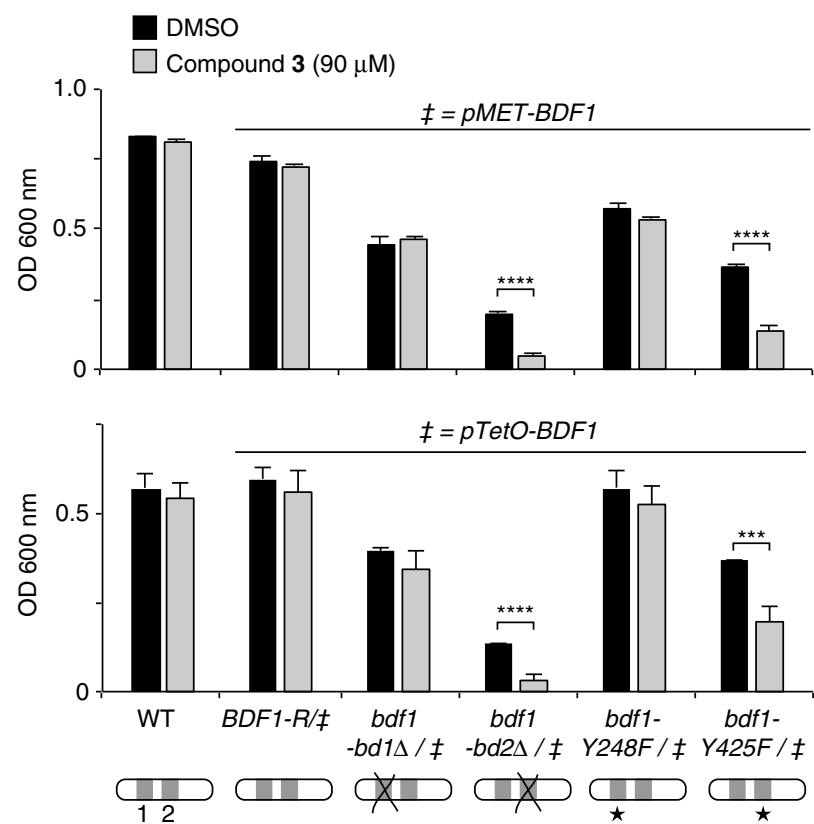

C
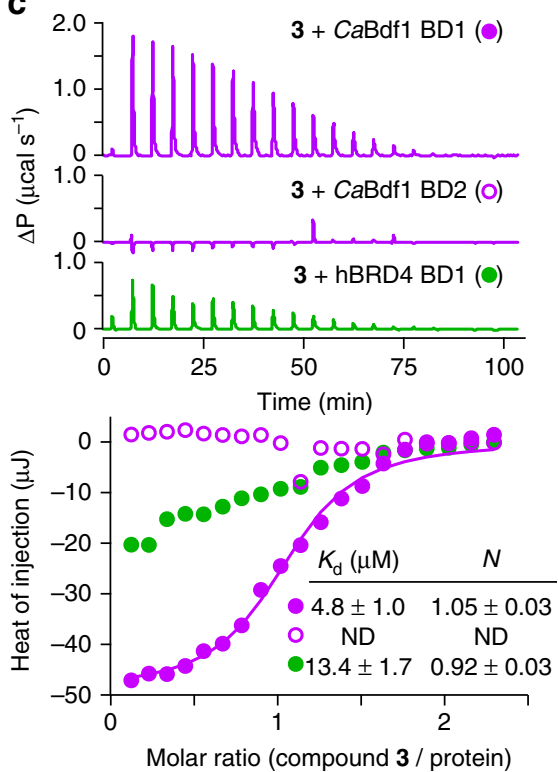

d
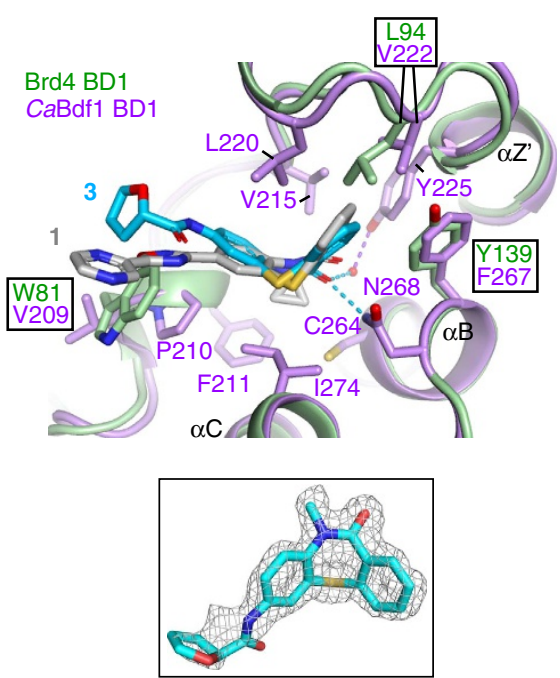

$\mathbf{f}$
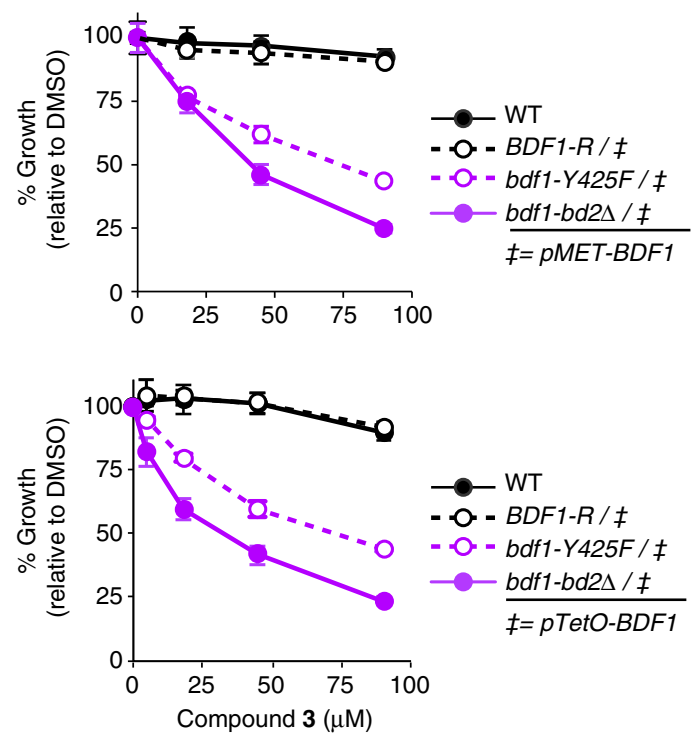

Figure 8 | A CaBdf1 BD1 inhibitor phenocopies the effect of BD1 inactivation on C. albicans growth. (a) Chemical structure of compound 3. (b) HTRF assay showing that $\mathbf{3}$ inhibits CaBdf1 BD1 preferentially over CaBdf1 BD2 and with modest selectivity over Brd4 BD1. (c) Representative ITC experiments showing the binding of $\mathbf{3}$ towards the indicated BD. The values indicated for $K_{d}$ and $N$ represent the mean and s.d. from three independent experiments. See also Supplementary Table 1. (d) Crystal structure of CaBdf1 BD1 (violet) bound to $\mathbf{3}$ (S enantiomer) and alignment with CaBdf1 BD1 bound to 1 and with human Brd4 BD1 (green). Side chains are shown for CaBdf1 residues in contact with $\mathbf{3}$ and for the corresponding Brd4 residues if divergent from CaBdf1. Inset, simulated-annealing omit $F_{\mathrm{o}}-F_{\mathrm{C}}$ density for compound $\mathbf{3}$ contoured at $3 \sigma$. (e) Compound $\mathbf{3}$ compromises the in vitro growth of $C$. albicans when CaBdf1 $\mathrm{BD} 2$ is inactivated by deletion or point mutation. Strains were grown in the presence of methionine/cysteine or Dox to repress expression of the WT allele from the $p M E T$ (top) or pTetO (bottom) promoter, respectively. Data represent mean and s.d. values from three independent experiments. ${ }^{\star \star \star} P \leq 0.01$; ${ }^{\star \star \star \star} P \leq 10^{-3}$. $P$ values were determined in a two-sided Welch $t$-test. (f) Dose-response experiments showing the effect of $\mathbf{3}$ on Bdf1 mutant strains. Met/Cys or doxycyline were added to repress expression from the pMET (top) or pTetO (bottom) promoter, respectively. Data represent mean and s.d. values from three independent experiments.

significant growth defect was detected. The most active compound was dibenzothiazepinone 3 , an analogue of $\mathbf{1}$ in which the cyclopropyl, oxadiazole and pyrazine groups are replaced by smaller methyl, carboxyamidyl and tetrahydrofuran groups, respectively (Fig. 8a). HTRF and ITC assays showed that 3 inhibits $C a B d f 1 B D 1$ with low-micromolar affinity and three- to fivefold selectivity relative to human Brd4 BD1 (Fig. 8b,c and
Supplementary Table 1). The crystal structure of CaBdf1 BD1 bound to 3 shows that the compound is slightly rotated within the binding pocket compared to 1 (Fig. 8d). Consequently, in a structural alignment with Brd4 BD1, the steric hindrance observed for 1 due to the larger Trp81 and Leu94 side chains is largely relieved for compound $\mathbf{3}$, explaining the reduced selectivity of this compound. (Both enantiomers of 3 are 
equally active, consistent with the lack of interaction between the $\mathrm{BD}$ and the tetrahydrofuran ring; Supplementary Fig. 9). Liquid culture assays show that 3 has little effect on the growth of C. albicans strains expressing either WT Bdf1 or Bdf1 inactivated in $\mathrm{BD} 1$, but significantly inhibited the growth of strains inactivated in BD2 (Fig. 8e), consistent with the specificity of compound 3 for BD1. A dose-response curve revealed a cellular $\mathrm{EC}_{50}$ value of $35 \pm 7 \mu \mathrm{M}$, corresponding to a 20 -fold cell shift relative to the $\mathrm{IC}_{50}$ value determined by $\mathrm{HTRF}$ in vitro (or sevenfold relative to the $K_{\mathrm{d}}$ by ITC) (Fig. 8f). At this concentration, compound 3 showed relatively little cytotoxicity towards mammalian cells $\left(\mathrm{EC}_{50}>100 \mu \mathrm{M}\right.$; Supplementary Fig. 6). Taken together, these results demonstrate the feasibility of using a small-molecule inhibitor to antagonize CaBdf1 function in the fungal cell.

\section{Discussion}

We have shown that $C$. albicans $B D F 1$ deletion is lethal and that mutations abolishing BD-mediated ligand-binding activity severely compromise growth (Fig. 2c-e), validating BET BD inhibition as a potential antifungal strategy in $C$. albicans. Whereas the combined inactivation of both CaBdf1 BDs is lethal, that of a single BD leads to only a partial reduction in growth (Fig. 2d,e and Fig. 8e). This likely reflects functional redundancy between the two BDs, consistent with their similar binding profiles towards acetylated H4 tails (Fig. 1d,e). Growth inhibition appears slightly more pronounced on BD2 inactivation compared to that of BD1 (Fig. 2d,e). This contrasts with the finding that the selective inhibition of human BET BD2 in liver cancer cells induced only minor effects on transcriptional regulation ${ }^{43}$ and that $\mathrm{BD} 1$ plays a more important role than $\mathrm{BD} 2$ in Brdt-mediated chromatin remodelling ${ }^{44}$, in recruiting $\mathrm{Brd} 3$ to acetylated sites on GATA1 (ref. 45), and in chromatin binding by Brd4 (ref. 46). This discrepancy may partly reflect differences between human and C. albicans BET BD selectivity towards acetylated histone peptides, as well as different histone acetylation patterns in these species.

Because C. albicans strains inactivated in only one Bdf1 BD remain viable, a chemotherapeutic strategy targeting $\mathrm{CaBdf1}$ would likely require the inhibition of both BDs to be effective. Such inhibition could be achieved via a single compound, which targets both BDs or through the combined use of two compounds, supplied independently or covalently linked as a bivalent $\mathrm{BD}$ inhibitor. Indeed, dual-warhead BET inhibitors that simultaneously engage both BDs within a single BET protein have recently been described, which possess greatly enhanced biochemical and cellular potency as well as increased efficacy in animal disease models relative to monovalent inhibitors ${ }^{47-49}$. Alternatively, one could envisage a Proteolysis Targeting Chimera approach, whereby a single BD inhibitor conjugated to a ligand for an E3 ubiquitin ligase would trigger the degradation of $\mathrm{CaBdf1}$, as recently demonstrated for human BET proteins ${ }^{50-53}$.

Compounds 1 and 2 target $\mathrm{CaBdf1} \mathrm{BD} 1$ and BD2, respectively, with low-micromolar affinity, yet do not significantly inhibit human BET or non-BET BDs, demonstrating that small-molecule inhibition of $C a B d f 1$ BDs can be achieved with high selectivity. The selectivity of $\mathbf{1}$ and $\mathbf{2}$ for $C a B d f 1$ results from steric incompatibility with bulkier side chains in the human BET BD-binding pockets, particularly at signature positions 1 and 3 . Remarkably, all $169 \mathrm{CaBdf1}$-selective hits identified in our screen target either $\mathrm{CaBdf1} \mathrm{BD} 1$ or BD2, but not both, in contrast with many BETi compounds, which inhibit both BD1 and BD2 in human BET proteins. A likely explanation is that, whereas residues at signature positions 1 and 3 are conserved between human BET BD1 and BD2, they diverge between CaBdf1 BD1 and BD2, facilitating the identification of $\mathrm{CaBdf1}$ inhibitors with single BD selectivity (Supplementary Fig. 10). Nevertheless, an inhibitor that targets both $\mathrm{CaBdf1}$ BDs without inhibiting human BET BDs is in principle achievable, because the WPF-shelf Trp residue at signature position 3 in human $\mathrm{BET} B \mathrm{BD}$ is bulkier than the corresponding ( $\mathrm{Val}$ and $\mathrm{Phe}$ ) residues in both $\mathrm{CaBdf1} \mathrm{BD} 1$ and BD2. For example, medicinal chemistry optimization of compound 2 could conceivably yield an analogue that would inhibit both $\mathrm{CaBdf1} \mathrm{BD} 1$ and $\mathrm{BD} 2$ without losing selectivity against human BET BDs.

Our chemical screen identified a dibenzothiazepinone compound, 3, that selectively inhibited CaBdf1 BD1 and displayed antifungal activity against susceptible strains. Although we were unable to obtain biochemical evidence for a direct interaction between 3 and Bdf1 in C. albicans cells, the different susceptibility of Bdf1 mutant strains provides compelling evidence that Bdf1 $\mathrm{BD} 1$ is the intracellular target (Fig. 8e,f). Specifically, strains expressing Bdf1 mutants in which BD1 was the lone functional BD (mutants bdf1-bd2 $\Delta$ and bdf1-Y425F) were sensitive to 3, consistent with this compound's selectivity for CaBdf1 BD1, whereas all other strains with a functional BD2 were insensitive, arguing strongly against a potential off-target effect. Strikingly, compound 1 did not significantly inhibit growth, despite being an analogue of 3 with nearly identical binding affinity for CaBdf1 BD1. This makes the dibenzothiazepinone an attractive scaffold for structure-activity relationship studies, as these may reveal the molecular determinants of a compound's ability to reach its intracellular target and thereby lead to analogues with improved cellular potency.

In conclusion, Bdf1 BDs are required for the viability and virulence of $C$. albicans and can be selectively targeted by smallmolecule inhibitors without compromising human BET BD function. These findings pave the way for the development of BET inhibitors as a novel class of antifungal therapeutics.

\section{Methods}

Chemicals. BET inhibitors PFI-1, IBET-151 and bromosporine were purchased from Sigma while JQ1 was from Clinisciences (Nanterre, France). Compound 1 was purchased from ChemDiv as powder and dissolved in dimethylsulfoxide (DMSO) without further purification. Compounds $\mathbf{2}$ and $\mathbf{3}$ (the latter as the racemate), initially purchased from ChemDiv, were resynthesized in our laboratory where the individual enantiomers of $\mathbf{3}$ were also prepared (Supplementary Methods) and used for all in vitro and cell-based assays. Derivatives $\mathbf{2 a}$ and $\mathbf{2 b}$ were synthesized in our laboratory for this study (Supplementary Methods).

Protein expression and purification. Proteins used for crystallization and for ITC. DNA fragments encoding CaBdf1 BD1 (residues 193-327) or BD2 (residues 386-491) and human Brd4 BD1 (residues 22-204) were PCR amplified from genomic or cDNA libraries and cloned into a pETM11 vector as fusion constructs bearing an N-terminal His tag followed by a tobacco etch virus (TEV) protease cleavage site. (Primer sequences are listed in Supplementary Table 4.) Transformed E. coli strain BL21(DE3) (New England Biolabs, ref. C2527I) cells were grown in LB medium containing kanamycin $\left(50 \mu \mathrm{g} \mathrm{ml}^{-1}\right)$ at $37^{\circ} \mathrm{C}$ until reaching an $\mathrm{OD}_{600}$ of 1 , induced with $0.5 \mathrm{mM}$ IPTG and further incubated at $16^{\circ} \mathrm{C}$ for $12-20 \mathrm{~h}$ before collecting. Cells were resuspended in buffer A $(50 \mathrm{mM}$ Tris- $\mathrm{HCl}$ pH 7.5, $300 \mathrm{mM}$ $\mathrm{NaCl}, 10 \%$ glycerol, $25 \mathrm{mM}$ imidazole, $5 \mathrm{mM} \beta$-mercaptoethanol and protease inhibitors) and lysed by sonication. The cleared lysate was incubated with Ni-NTA resin (Qiagen) and washed with buffer A containing $0.5 \mathrm{M} \mathrm{NaCl}$. Proteins were eluted with $250 \mathrm{mM}$ imidazole, dialysed overnight in the presence of His-tagged TEV protease against buffer A containing no imidazole and incubated with $\mathrm{Ni}$ NTA resin to remove His-tagged species. Proteins were further purified on a Superdex 75 10/300 (GE Healthcare) column in $50 \mathrm{mM}$ Hepes pH 7.5, $150 \mathrm{mM}$ $\mathrm{NaCl}, 0.5 \mathrm{mM}$ dithiothreitol (DTT). Proteins were concentrated to $>20 \mathrm{mg} \mathrm{ml}^{-1}$ on a Centricon device (Millipore) and used for ITC, TSA and crystallization experiments. Human SMARCA2 and SMARCA4 BD plasmids bearing an $\mathrm{N}$-terminal His tag and TEV protease site were purchased from Addgene (plasmid no. 73250 and 74664 , respectively). Expression and purification were performed as above, except that buffer A was replaced by buffer B (50 mM Hepes $\mathrm{pH} 7.5$, $500 \mathrm{mM} \mathrm{NaCl}, 5 \%$ glycerol, $5 \mathrm{mM}$ imidazole and protease inhibitors) and gel filtration was performed in $10 \mathrm{mM}$ Hepes $\mathrm{pH} 7.5,500 \mathrm{mM} \mathrm{NaCl}$ and $5 \%$ glycerol.

Proteins used for HTRF assays. GST-tagged human Brd4 BD2 (residues

349-460) was purchased from Reaction Biology Corp. Human Brd4 BD1 (residues 
22-204) and CaBdf1 BD1 (residues 193-327) and BD2 (residues 361-501) were cloned into a pGEX4t1 vector as GST-tagged fusion proteins. Expression in E. coli strain BL21(DE3) cells was performed as for His-tagged constructs. Collected cells resuspended in $50 \mathrm{mM}$ Tris- $\mathrm{HCl} \mathrm{pH} 7.5,150 \mathrm{mM} \mathrm{NaCl}$ and protease inhibitors were lysed by sonication. The clarified lysate was incubated with glutathione sepharose (GE Healthcare) and then washed with $50 \mathrm{mM}$ Tris- $\mathrm{HCl} \mathrm{pH} \mathrm{7.5,}$ $500 \mathrm{mM} \mathrm{NaCl}$ and $1 \% \mathrm{NP}-40$. Proteins were eluted with $10 \mathrm{mM}$ glutathione and further purified on a Superdex 75 10/300 (GE Healthcare) column in $50 \mathrm{mM}$ Hepes pH 7.5, $150 \mathrm{mM} \mathrm{NaCl}, 0.5 \mathrm{mM}$ DTT.

Pull-down and peptide array assays. Biotinylated peptides corresponding to non-acetylated (H4ac0) and tetra-acetylated (H4K5acK8acK12acK16ac) histone $\mathrm{H} 4$ tails were synthesized by Covalab (Villeurbanne, France) and immobilized on Streptavidin-coated magnetic beads (Dynabeads MyOne Streptavidin C1; Thermo Fisher) according to the manufacturer's instructions. Beads were incubated with $1.25 \mu \mathrm{g}$ of GST-tagged CaBdf1 BD1 or BD2 in binding buffer $(50 \mathrm{mM}$ Tris $\mathrm{pH} 7.4$, $150 \mathrm{mM} \mathrm{NaCl}, 0.1 \%, \mathrm{NP}-40,10 \%$ glycerol $10 \%, 1 \mathrm{mM}$ DTT) in a volume of $250 \mu \mathrm{l}$ for $2 \mathrm{~h}$ at $4{ }^{\circ} \mathrm{C}$ and subsequently washed in binding buffer containing $500 \mathrm{mM}$ $\mathrm{NaCl}$. Bound proteins were eluted by boiling in SDS-PAGE sample loading buffer and analysed by western blot using an anti-GST antibody (GE Healthcare).

Histone peptide arrays were obtained from Active Motif (ref. 13005) and used in accordance with the supplier's recommendations. In short, arrays were blocked and incubated with $1 \mu \mathrm{M}$ of purified CaBdf1 BD1 or BD2. Arrays were incubated with recombinant $\mathrm{BDs}$ for $2 \mathrm{~h}$ at $4{ }^{\circ} \mathrm{C}$ in binding buffer (Tris- $\mathrm{HCl} 50 \mathrm{mM} \mathrm{pH}$ 7.4, NP-40 $0.1 \%, \mathrm{NaCl} 150 \mathrm{mM}$, glycerol $10 \%$ ). They were then washed in the same buffer and binding detected by a standard western blot procedure using an antiGST antibody (Dutscher, ref. 27-4577-01). An antibody directed against a spotted c-myc peptide (supplied with the MODified Array Labeling Kit (Active Motif, ref. 13006) and used following the manufacturers' instructions at 1/2,000 dilution) was used as a positive control and its signal intensity was used for normalization. Assays were performed in duplicate. Images were acquired on a Bio-Rad ChemiDoc XRS Imaging machine and data were quantified using the array analyser software provided by Active Motif. The results of the quantification are summarized in Supplementary Data 1.

ITC. Calorimetric experiments were performed on a NanoITC calorimeter (TA Instruments) at $25^{\circ} \mathrm{C}$ while stirring at 250 r.p.m. All proteins were buffer exchanged by gel filtration into $50 \mathrm{mM}$ Hepes $\mathrm{pH} 7.5,150 \mathrm{mM} \mathrm{NaCl}, 0.5 \mathrm{mM}$ DTT, except for SMARCA2 and SMARCA4A BDs, which were exchanged into $10 \mathrm{mM}$ Hepes pH 7.5, $500 \mathrm{mM} \mathrm{NaCl}$ and 5\% glycerol. Typically, 10-100 $\mu \mathrm{M}$ compound and $60-900 \mu \mathrm{M}$ protein were placed in the cell and syringe, respectively. Titrations consisted of an initial injection of $1.5 \mu \mathrm{l}$ followed by 19 identical injections of $2.5 \mu \mathrm{l}$ made at time intervals of $5 \mathrm{~min}$. ITC data were corrected for the heat of dilution of injectant into buffer and analysed with software provided by the manufacturer using a single binding site model. The first data point was excluded from the analysis. Thermodynamic parameters determined from the ITC data are summarized in Supplementary Table 1.

HTRF assay. Biotinylated and non-biotinylated tetra-acetylated $\mathrm{H} 4$ peptides (H4K5acK8acK12acK16ac; denoted H4ac4) were synthetized by Covalab (Villeurbanne, France). HTRF reagents and buffers were purchased from Cisbio Bioassays and the assay performed according to the manufacturer's instructions. Specifically, the assay used a terbium(III) cryptate donor reagent conjugated to an anti-GST antibody (MAb anti-GST-Tb; ref. 61GSTTLA), a streptavidin-conjugated acceptor reagent (streptavidin-d2; ref. 610SADLA) and Cisbio proprietary buffers (EPIgeneous Binding Domain Diluent and Detection buffer; refs. 62DLBDDF and 62DB2FDG, respectively). Incubation with GST-tagged BDs and biotinylated H4ac4 brings the donor and acceptor into close proximity and allows FRET. The non-biotinylated H4ac4 peptide competes for binding and was used as a positive control for inhibition. GST-tagged proteins in $25 \mathrm{mM}$ Hepes pH 7.5, $150 \mathrm{mM}$ $\mathrm{NaCl}, 0.5 \mathrm{mM}$ DTT were assayed at $5 \mathrm{nM}$ final concentration. Biotinylated $\mathrm{H} 4 \mathrm{ac} 4$ peptides were used at a final concentration of $50,600,300$ or $400 \mathrm{nM}$ in assays involving Brd4 BD1, Brd4 BD2, CaBdf1 BD1 and CaBdf1 BD2, respectively. The antibody-conjugated donor was used at $0.5 \mathrm{nM}$ and the streptavidin-conjugated acceptor was used at $1 / 8$ of the H4ac4 peptide concentration. Inhibitors were tested by performing a nine-point dilution series with a maximal final concentration of $10 \mu \mathrm{M}$ (JQ1, IBET-151, PFI-1, bromosporine), $20 \mu \mathrm{M}(\mathbf{1 , 3})$ or $40 \mu \mathrm{M}$ (2). These concentrations allowed the DMSO concentration to remain below the limit ( $1 \%$ for $\mathrm{CaBdf1} \mathrm{BD} 1$ and Brd4 BD1, and 0.4\% for $\mathrm{CaBdf1} \mathrm{BD} 2$ and Brd4 BD2) that allowed the assay to maintain a $Z^{\prime}$ factor $^{54} \geq 0.8$. Components were incubated at room temperature for $1 \mathrm{~h}(\mathrm{CaBdf} 1 \mathrm{BD} 1$ and $\mathrm{Brd} 4 \mathrm{BD} 1)$ or at $4{ }^{\circ} \mathrm{C}$ for $6-24 \mathrm{~h}(\mathrm{CaBdf} 1$ $\mathrm{BD} 2$ and Brd4 BD2). Experiments were performed in 384-well white plates (Greiner 781080) in a volume of $20 \mu \mathrm{l}$ and analysed in a ClarioStar plate reader (BMG LABTECH). Excitation was at $330 \mathrm{~nm}$ and emission intensities were measured at 620 and $665 \mathrm{~nm}$ (corresponding to the donor and acceptor emission peaks, respectively; the $665 / 620$ ratio is used to calculate the specific HTRF signal) with an integration delay of $60 \mu \mathrm{s}$ and an integration time of $400 \mu \mathrm{s}$.
High-throughput chemical screening. The HTRF assay described above was miniaturized for performance in a $5 \mu \mathrm{l}$ reaction volume in 1,536-well black plates. Approximately 80,000 compounds comprising the soluble diversity (ChemDiv), targeted diversity (ChemDiv) and $30 \mathrm{~K}$ diversity (LifeChem) collections were dispensed into wells by an Echo acoustic liquid dispenser. A master mix comprising $\mathrm{MAb}$ anti-GST-Tb donor, streptavidin-d2 acceptor, GST-tagged BD protein, biotinylated H4ac4 peptide was then added and the plates incubated for $6-24 \mathrm{~h}$ prior to reading. The primary screen was performed with compounds at a final concentration of $20 \mu \mathrm{M}$ (CaBdf1 BD1) or $8 \mu \mathrm{M}$ (CaBdf1 BD2), corresponding to a final DMSO concentration of $1 \%$ and $0.4 \%$, respectively. Hits were initially confirmed by repeating the assay at a single concentration in triplicate, and subsequently by dose-response curves constructed using eight-point dilutions between 0 and 20 (or 8 ) $\mu \mathrm{M}$.

BROMOscan profiling. BROMOscan profiling was performed by DiscoverX (Fremont, CA, USA). T7 phage strains displaying BDs were grown in parallel 24-well blocks in an E. coli host derived from the BL21 strain as described ${ }^{55}$. E. coli were grown to log-phase and infected with T7 phage from a frozen stock (multiplicity of infection $=0.4$ ) and incubated with shaking at $32{ }^{\circ} \mathrm{C}$ until lysis (90-150 $\mathrm{min})$. The lysates were centrifuged $(5,000 \mathrm{~g})$ and filtered $(0.2 \mu \mathrm{m})$ to remove cell debris. Streptavidin-coated magnetic beads were treated with biotinylated small molecule or acetylated peptide ligands for $30 \mathrm{~min}$ at room temperature to generate affinity resins for $\mathrm{BD}$ assays. Ligand-loading densities were optimized as described ${ }^{56}$. The liganded beads were blocked with excess biotin and washed with blocking buffer (SeaBlock (Pierce), 1\% BSA, 0.05\% Tween 20, $1 \mathrm{mM}$ DTT) to remove unbound ligand and to reduce non-specific phage binding. Binding reactions were assembled by combining $\mathrm{BDs}$, liganded affinity beads and test compounds in $1 \times$ binding buffer ( $16 \%$ SeaBlock, $0.32 \times$ PBS, $0.02 \%$ BSA, $0.04 \%$ Tween $20,7.9 \mathrm{mM}$ DTT). Test compounds were prepared as $1,000 \times$ stocks in 100\% DMSO and subsequently diluted 1:25 in monoethylene glycol. The compounds were then diluted directly into the assays such that the final concentrations of DMSO and monoethylene glycol were $0.1 \%$ and $2.4 \%$, respectively. All reactions were performed in polypropylene 384 -well plates in a final volume of $20 \mu \mathrm{l}$. The assay plates were incubated at room temperature with shaking for $1 \mathrm{~h}$ and the affinity beads were washed with wash buffer $(1 \times \mathrm{PBS}$, $0.05 \%$ Tween 20$)$. The beads were then resuspended in elution buffer $(1 \times$ PBS, $0.05 \%$ Tween $20,2 \mu \mathrm{M}$ non-biotinylated affinity ligand) and incubated at room temperature with shaking for $30 \mathrm{~min}$. The $\mathrm{BD}$ concentration in the eluates was determined by qPCR. Compounds $\mathbf{1}$ and $\mathbf{2}$ were screened at $10 \mu \mathrm{M}$ concentration in duplicate. DMSO was used as a negative control and a small-molecule or peptide ligand specific for each $\mathrm{BD}$ in the screen was used as a positive control. Results are reported as percent control, where lower values indicate stronger inhibition. Percent control was calculated as $100 \times(t-p) /(n-p)$, where $t$ is the test compound signal and $p$ and $n$ are the positive and negative control signals, respectively.

Crystallization and crystal structure determination. Initial crystallization conditions were identified by the sitting drop vapour diffusion method at $4{ }^{\circ} \mathrm{C}$ using a Cartesian PixSys 4200 crystallization robot at the high-throughput crystallization laboratory of the EMBL Grenoble Outstation (https://htxlab.embl.fr). Crystals used for data collection were obtained by the hanging drop method at $4{ }^{\circ} \mathrm{C}$ by mixing $1 \mu \mathrm{l}$ of the protein or protein/inhibitor sample with $1 \mu \mathrm{l}$ of the reservoir solution, as follows. Unbound CaBdf1 BD1 $\left(40 \mathrm{mg} \mathrm{ml}^{-1}\right)$ was mixed with $0.2 \mathrm{M}$ ammonium iodide (pH 6.5) and 26\% (w/v) PEG 3350. Unbound CaBdf1 BD2 (40 $\left.\mathrm{mg} \mathrm{ml}^{-1}\right)$ was mixed with $0.1 \mathrm{M}$ Tris- $\mathrm{HCl}(\mathrm{pH} 8.5)$ and $1 \mathrm{M}$ ammonium phosphate. CaBdf1 BD1 bound to compound 1 was crystallized by mixing a solution of $20 \mathrm{mg} \mathrm{ml}^{-1}$ protein and $0.4 \mathrm{mM}$ inhibitor with $0.1 \mathrm{M}$ Tris- $\mathrm{HCl}(\mathrm{pH} 8.5), 23 \%$ (w/v) PEG MME 2000 and $10 \mathrm{mM} \mathrm{NiCl}_{2}$. CaBdf1 BD1 bound to compound 3 was crystallized by mixing a solution of $25 \mathrm{mg} \mathrm{ml}^{-1}$ protein and $1.5 \mathrm{mM}$ inhibitor with $0.1 \mathrm{M}$ sodium acetate (pH 4.6) and $2.4 \mathrm{M}$ ammonium sulfate. CaBdf1 BD2 bound to compound 2 was crystallized by mixing a solution of $22 \mathrm{mg} \mathrm{ml}^{-1}$ protein and $1.5 \mathrm{mM}$ inhibitor with $0.1 \mathrm{M}$ sodium acetate ( $\mathrm{pH} 4.6$ ) and 25\% (w/v) PEG 3000. Crystals were flashcooled in liquid nitrogen after being transferred into the well solution supplemented with the following cryo-protectants: unbound $\mathrm{CaBdf1} \mathrm{BD} 1$, unbound CaBdf1 BD2 and CaBdf1 BD1 bound to 1, 30\% (v/v) glycerol; CaBdf1 BD1 bound to 3(S), 3.2 M ammonium sulfate; CaBdf1 BD2 bound to $2,25 \%$ (v/v) glycerol.

Diffraction data were collected at beamlines of the European Synchrotron Radiation Facility (ESRF), as indicated in Supplementary Table 2. Data collected on MarMOSAIC (ID23-2), Pilatus 6M (ID29) and Pilatus3 2M (ID30A-1) detectors were processed using X-ray Detector Software $(\mathrm{XDS})^{57}$ and programmes of the CCP4 suite ${ }^{58}$. Structures were solved by molecular replacement using Phaser ${ }^{59}$ Structures 2OSS and 2OUO (human Brd4 BD1 and BD2, respectively) were used as search models to solve the structures of $\mathrm{CaBdf1} \mathrm{BD} 1$ and $\mathrm{BD} 2$, respectively. Initial solutions were improved by automated protein chain tracing with ARP/WARP ${ }^{60}$ followed by further manual building using $\mathrm{Coot}^{61}$. Inhibitor coordinates and .cif restraints files were generated using the Cactus online SMILES translator (https://cactus.nci.nih.gov/translate/) and JLigand ${ }^{62}$, respectively. Structures were refined with Phenix ${ }^{63}$ and validated using MolProbity ${ }^{64}$ and the built-in tools in Coot. Examples of final $2 F_{\mathrm{o}}-F_{\mathrm{c}}$ electron density are provided in Supplementary Figs 11 and 12. Data collection and refinement statistics are summarized in Supplementary Table 2. 
Cytotoxicity assays on human cells. Proliferation of human cells was assessed using an MTT colorimetric assay (Cell Proliferation Kit I, Roche). HeLa (epithelial cells, ATCC number CCL-2) and IMR90 (primary fibroblasts cells, ATCC number CCL-186) cells were cultured in humidified atmosphere $\left(37^{\circ} \mathrm{C}\right.$ and $5 \% \mathrm{CO}_{2}$ ) in DMEM medium containing $10 \%$ heat inactivated foetal calf serum and $2 \mathrm{mM}$ glutamine. Cells were seeded at a concentration of 5,000 HeLa cells per well or 15,000 IMR90 cells per well in $100 \mu \mathrm{l}$ culture medium containing the test compound (compounds $\mathbf{1 , 2 , 3}$, amphotericin B or fluconazole) into 96 wells microplates (Falcon ref. 353072). Plates were incubated at $37^{\circ} \mathrm{C}$ and $5 \% \mathrm{CO}_{2}$ for $28 \mathrm{~h}$ before adding $10 \mu \mathrm{l}$ of MTT labelling reagent (final concentration $0.5 \mathrm{mg} \mathrm{ml}^{-1}$ ) to each well. After incubating for a further $4 \mathrm{~h}, 100 \mu \mathrm{l}$ of the solubilization solution were added in each well. Plates were allowed to stand overnight in the incubator before measuring the spectrophotometrical absorbance at $570 \mathrm{~nm}$ and at the reference wavelength of $690 \mathrm{~nm}$ in a ClarioStar plate reader. The values

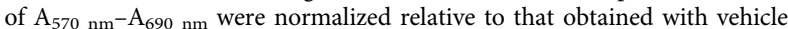
$(0.2 \% \mathrm{DMSO})$ and plotted against compound concentration.

\section{Generation of $\mathbf{C}$. albicans mutant strains. Plasmids used in this study to} incorporate different mutations into the BDF1 gene are listed in Supplementary Table 5. All DNA fragments were fused in a pCR2.1-TOPO vector using a Gibson assembly kit (New England Biolabs) and validated by sequencing. The pMET3 promoter sequence was recycled from the plasmid pFA-ARG4-MET3p (ref. 65). The pTetO cassette (Fig. 2a) was generated by assembling the following fragments with the Gibson assembly kit (New England Biolabs) in a pCR2.1-TOPO vector: (i) ARG4 gene from the plasmid pFA-ARG4-MET3p (ref. 65); (ii) a Tet-dependenttransactivation protein (TetR-VP16) obtained by gene synthesis with corrections for the genetic code of the CTU clade (Thermo), flanked by the promoter (500 bp) and terminator (500 bp) of the C. albicans TDH 3 gene; (iii) the repressible Tet-operator (pTetO) from the plasmid pCM184 (ref. 66). In this study, $500 \mathrm{bp}$ homologous regions were used to integrate the pTet cassette upstream of the BDF1 ORF. The BDF1 point mutant cassettes were obtained using the QuikChange sitedirected kit (Agilent) with the BDF1 plasmid pJG214 or pJG215. All cassettes were obtained by digesting $4 \mu \mathrm{g}$ of the pCR2.1-TOPO plasmids and were transformed in C. albicans by a lithium acetate procedure, as previously described ${ }^{67}$. Mutations introduced in the BDF1 gene in C. albicans were confirmed by PCR, sequencing of the mutated region in the genomic DNA and western blot. C. albicans strains used in this study are listed in Supplementary Table 6.

Growth assays. Growth on solid media. C. albicans strains were grown in SC media to an $\mathrm{OD}_{600}$ of $0.5-0.8$, pelleted and resuspended in sterile water at a final $\mathrm{OD}_{600}$ of 0.13 . Cells were spotted on solid media in a threefold dilution series starting at an $\mathrm{OD}_{600}$ of 0.13 . Plates were incubated at $30^{\circ} \mathrm{C}$ for 1 day before imaging. Stress resistance was tested using the following conditions: $\mathrm{H}_{2} \mathrm{O}_{2}(4.5$ and $6 \mathrm{mM})$, methylmethanesulfonate $(0.005$ and $0.02 \%)$, hydroxyurea $(10 \mathrm{mM})$, nicotinamide $(0.5,1$, and $4 \mathrm{mM})$, caffeine $(15 \mathrm{mM})$, sorbitol $(1,1.5$ and $2 \mathrm{M})$, Congo red $\left(50 \mu \mathrm{g} \mathrm{ml}^{-1}\right)$, calcofluor white $(20 \mu \mathrm{M})$, SDS $(0.04 \%)$ and heat shock at $42^{\circ} \mathrm{C}$.

Growth on liquid media. C. albicans strains were grown at $30^{\circ} \mathrm{C}$ in $\mathrm{SC}$ medium to an $\mathrm{OD}_{600}$ of $0.5-0.8$. For the evaluation of growth defects related to Bdf1 mutations, log-phase growing cells were counted using a Neubauer chamber and diluted in liquid media to a final concentration of 5,400 cells per $\mathrm{ml}$ per well in a sterile 96 -well plate. Dox $\left(50 \mu \mathrm{g} \mathrm{ml}^{-1}\right)$ or methionine $(5 \mathrm{mM}) /$ cysteine $(0.25 \mathrm{mM})$ were added to the media if required. Plates were incubated at $30^{\circ} \mathrm{C}$ and $\mathrm{OD}_{600}$ was measured using a Multiskan FC Microplate Photometer (Thermo Fisher). For the evaluation of chemical compounds, strains were grown in SC medium supplemented with $50 \mu \mathrm{g} \mathrm{ml}^{-1}$ Dox or $5 \mathrm{mM}$ methionine $/ 0.25 \mathrm{mM}$ cysteine $24 \mathrm{~h}$ before counting and then seeded in 96-well plates at the same concentration as mentioned above, with or without chemical compounds. Statistical tests were performed using two-sided Welch $t$-tests.

Analysis of whole-cell extracts and antibodies. C. albicans strains were grown at $30^{\circ} \mathrm{C}$ in SC media to an $\mathrm{OD}_{600}$ of $0.5-0.8$. Cells were lysed in FastprepTM (MPBiologicals) twice at $6.5 \mathrm{~m} \mathrm{~s}^{-1}$ for $1 \mathrm{~min}$ with intermediate incubation on ice. Histone H4 antibody was purchased from Active Motif (ref. 39269) and the CaBdf1 antibody was developed in house using a full-length recombinant protein injected in rabbits (Covalab).

Mouse experiments. A mouse model of hematogenously disseminated candidiasis was used, as approved by the French National Animal Experimentation Ethics Committee (reference B385161006) under the file reference 2015042015538706 v4 (AFAPIS\#644). Candida strains were inoculated from an overnight subculture in liquid yeast extract peptone dextrose (YPD). In each condition, six female BALB/c mice ( 8 weeks old) were injected in the lateral caudal vein with $2 \times 10^{5}$ cells of a $C$. albicans suspension. If necessary, Dox repression was added to the drinking water starting at $48 \mathrm{~h}$ before the injection until the day of killing. To limit severity and duration of pain, a pilot experiment established an end point 7 days post infection, when the virulence of each strain was assessed by kidney fungal burden evaluation, as described before ${ }^{68,69}$. Briefly, mice were killed, left kidneys were removed aseptically and weighed, and homogenized suspensions were cultured to evaluate colony-forming units (CFU). The final results were expressed as log CFUs per gram of tissue and statistically tested using two-sided Wilcoxon rank sum tests with continuity correction.

Statistics. Each statistical test is indicated in the legend of the corresponding figure and has been chosen for its compliance with the sample size. Sample size for mouse experiments was chosen as the best compromise between statistical power and regulations of the French National Animal Experimentation Ethic Committee No inclusion/exclusion criteria were pre-established. Animal studies were neither randomized nor performed blinded.

Data availability. Coordinates and structure factors have been deposited in the Protein Data Bank with accession codes 5N15 (unbound CaBdf1 BD1), 5N16 (CaBdf1 BD1 bound to 1), 5N17 (CaBdf1 BD1 bound to 3), 5N13 (unbound CaBdf1 BD2) and 5N18 (CaBdf1 BD2 bound to 2). The authors declare that all other data supporting the findings of this study are available within the article and its Supplementary Information files, or from the corresponding authors on request.

\section{References}

1. Brown, G. D. et al. Hidden killers: human fungal infections. Sci. Transl. Med. 4, $165 r v 113$ (2012).

2. Moran, C., Grussemeyer, C. A., Spalding, J. R., Benjamin, Jr D. K. \& Reed, S. D. Candida albicans and non-albicans bloodstream infections in adult and pediatric patients: comparison of mortality and costs. Pediatr. Infect. Dis. J 28, 433-435 (2009).

3. Arendrup, M. C. Epidemiology of invasive candidiasis. Curr. Opin. Crit. Care 16, 445-452 (2010)

4. Maubon, D., Garnaud, C., Calandra, T., Sanglard, D. \& Cornet, M. Resistance of Candida spp. to antifungal drugs in the ICU: where are we now? Intensive Care Med. 40, 1241-1255 (2014).

5. Denning, D. W. \& Bromley, M. J. Infectious disease. How to bolster the antifungal pipeline. Science 347, 1414-1416 (2015).

6. Sanglard, D. Emerging threats in antifungal-resistant fungal pathogens. Front Med. 3, 11 (2016).

7. Hnisz, D., Tscherner, M. \& Kuchler, K. Targeting chromatin in fungal pathogens as a novel therapeutic strategy: histone modification gets infectious. Epigenomics 3, 129-132 (2011).

8. Garnaud, C., Champleboux, M., Maubon, D., Cornet, M. \& Govin, J. Histone deacetylases and their inhibition in Candida species. Front. Microbiol. 7, 1238 (2016).

9. Kmetzsch, L. Histone deacetylases: targets for antifungal drug development Virulence 6, 535-536 (2015).

10. Wurtele, H. et al. Modulation of histone $\mathrm{H} 3$ lysine 56 acetylation as an antifungal therapeutic strategy. Nat. Med. 16, 774-780 (2010).

11. Nishikawa, J. L. et al. Inhibiting fungal multidrug resistance by disrupting an activator-mediator interaction. Nature 530, 485-489 (2016).

12. Filippakopoulos, P. \& Knapp, S. Targeting bromodomains: epigenetic readers of lysine acetylation. Nat. Rev. Drug Discov. 13, 337-356 (2014).

13. Moriniere, J. et al. Cooperative binding of two acetylation marks on a histone tail by a single bromodomain. Nature 461, 664-668 (2009).

14. Filippakopoulos, P. et al. Histone recognition and large-scale structural analysis of the human bromodomain family. Cell 149, 214-231 (2012).

15. Nicodeme, E. et al. Suppression of inflammation by a synthetic histone mimic. Nature 468, 1119-1123 (2010).

16. Filippakopoulos, P. et al. Selective inhibition of BET bromodomains. Nature 468, 1067-1073 (2010).

17. Schaefer, U. Pharmacological inhibition of bromodomain-containing proteins in inflammation. Cold Spring Harb. Perspect. Biol. 6:a018671, 1-4 (2014).

18. Brand, M. et al. Small molecule inhibitors of bromodomain-acetyl-lysine interactions. ACS Chem. Biol. 10, 22-39 (2015).

19. Wang, C. Y. \& Filippakopoulos, P. Beating the odds: BETs in disease. Trends Biochem. Sci. 40, 468-479 (2015).

20. Ferri, E., Petosa, C. \& McKenna, C. E. Bromodomains: structure, function and pharmacology of inhibition. Biochem. Pharmacol. 106, 1-18 (2016).

21. Romero, F. A. et al. Disrupting acetyl-lysine recognition: progress in the development of bromodomain inhibitors. J. Med. Chem. 59, 1271-1298 (2016).

22. Ladurner, A. G., Inouye, C., Jain, R. \& Tjian, R. Bromodomains mediate an acetyl-histone encoded antisilencing function at heterochromatin boundaries. Mol. Cell 11, 365-376 (2003).

23. Matangkasombut, O. \& Buratowski, S. Different sensitivities of bromodomain factors 1 and 2 to histone H4 acetylation. Mol. Cell 11, 353-363 (2003).

24. Garcia-Oliver, E. et al. Bdf1 bromodomains are essential for meiosis and the expression of meiotic-specific genes. PLoS Genet. 13, e1006541 (2017).

25. Matangkasombut, O., Buratowski, R. M., Swilling, N. W. \& Buratowski, S. Bromodomain factor 1 corresponds to a missing piece of yeast TFIID. Genes Dev. 14, 951-962 (2000).

26. Krogan, N. J. et al. A Snf2 family ATPase complex required for recruitment of the histone H2A variant Htz1. Mol. Cell 12, 1565-1576 (2003). 
27. Mizuguchi, G. et al. ATP-driven exchange of histone $\mathrm{H} 2 \mathrm{AZ}$ variant catalyzed by SWR1 chromatin remodeling complex. Science 303, 343-348 (2004).

28. Govin, J. et al. Systematic screen reveals new functional dynamics of histones H3 and H4 during gametogenesis. Genes Dev. 24, 1772-1786 (2010).

29. Fu, J. et al. The yeast BDF1 regulates endocytosis via LSP1 under salt stress. Curr. Microbiol. 70, 671-678 (2015).

30. Liu, X. et al. Genetic and comparative transcriptome analysis of bromodomain factor 1 in the salt stress response of Saccharomyces cerevisiae. Curr. Microbiol. 54, 325-330 (2007)

31. Durant, M. \& Pugh, B. F. NuA4-directed chromatin transactions throughout the Saccharomyces cerevisiae genome. Mol. Cell Biol. 27, 5327-5335 (2007).

32. Wapinski, I., Pfeffer, A., Friedman, N. \& Regev, A. Natural history and evolutionary principles of gene duplication in fungi. Nature 449, 54-61 (2007).

33. Roemer, T. et al. Large-scale essential gene identification in Candida albicans and applications to antifungal drug discovery. Mol. Microbiol. 50, 167-181 (2003).

34. Xu, Q. R. et al. Molecular genetic techniques for gene manipulation in Candida albicans. Virulence 5, 507-520 (2014).

35. Dawson, M. A. et al. Inhibition of BET recruitment to chromatin as an effective treatment for MLL-fusion leukaemia. Nature 478, 529-533 (2011).

36. Fish, P. V. et al. Identification of a chemical probe for bromo and extra C-terminal bromodomain inhibition through optimization of a fragmentderived hit. J. Med. Chem. 55, 9831-9837 (2012).

37. Douglas, L. M. \& Konopka, J. B. Plasma membrane organization promotes virulence of the human fungal pathogen Candida albicans. J. Microbiol. 54, 178-191 (2016).

38. Francois, J. M. Cell surface interference with plasma membrane and transport processes in yeasts. Adv. Exp. Med. Biol. 892, 11-31 (2016).

39. Prasad, R., Rawal, M. K. \& Shah, A. H. Candida efflux ATPases and antiporters in clinical drug resistance. Adv. Exp. Med. Biol. 892, 351-376 (2016).

40. Cowen, L. E., Sanglard, D., Howard, S. J., Rogers, P. D. \& Perlin, D. S. Mechanisms of antifungal drug resistance. Cold Spring Harb. Perspect. Med. 5, a019752 (2014).

41. Chung, C. W. et al. Discovery and characterization of small molecule inhibitors of the BET family bromodomains. J. Med. Chem. 54, 3827-3838 (2011).

42. Vidler, L. R., Brown, N., Knapp, S. \& Hoelder, S. Druggability analysis and structural classification of bromodomain acetyl-lysine binding sites. J. Med. Chem. 55, 7346-7359 (2012).

43. Picaud, S. et al. RVX-208, an inhibitor of BET transcriptional regulators with selectivity for the second bromodomain. Proc. Natl Acad. Sci. USA 110, 19754-19759 (2013).

44. Pivot-Pajot, C. et al. Acetylation-dependent chromatin reorganization by BRDT, a testis-specific bromodomain-containing protein. Mol. Cell Biol. 23, 5354-5365 (2003)

45. Lamonica, J. M. et al. Bromodomain protein Brd3 associates with acetylated GATA1 to promote its chromatin occupancy at erythroid target genes. Proc. Natl Acad. Sci. USA 108, E159-E168 (2011).

46. Baud, M. G. et al. Chemical biology. A bump-and-hole approach to engineer controlled selectivity of BET bromodomain chemical probes. Science 346, 638-641 (2014).

47. Tanaka, M. et al. Design and characterization of bivalent BET inhibitors. Nat. Chem. Biol. 12, 1089-1096 (2016).

48. Waring, M. J. et al. Potent and selective bivalent inhibitors of BET bromodomains. Nat. Chem. Biol. 12, 1097-1104 (2016).

49. Rhyasen, G. W. et al. AZD5153: a novel bivalent BET bromodomain inhibitor highly active against hematologic malignancies. Mol. Cancer Ther. 15, 2563-2574 (2016).

50. Winter, G. E. et al. Drug development. Phthalimide conjugation as a strategy for in vivo target protein degradation. Science 348, 1376-1381 (2015).

51. Lu, J. et al. Hijacking the E3 ubiquitin ligase cereblon to efficiently target BRD4. Chem. Biol. 22, 755-763 (2015)

52. Zengerle, M., Chan, K. H. \& Ciulli, A. Selective small molecule induced degradation of the BET bromodomain protein BRD4. ACS Chem. Biol. 10, 1770-1777 (2015)

53. Raina, K. et al. PROTAC-induced BET protein degradation as a therapy for castration-resistant prostate cancer. Proc. Natl Acad. Sci. USA 113, 7124-7129 (2016).

54. Zhang, J. H., Chung, T. D. \& Oldenburg, K. R. A simple statistical parameter for use in evaluation and validation of high throughput screening assays. J. Biomol. Screen 4, 67-73 (1999).

55. Fabian, M. A. et al. A small molecule-kinase interaction map for clinical kinase inhibitors. Nat. Biotechnol. 23, 329-336 (2005).

56. Wodicka, L. M. et al. Activation state-dependent binding of small molecule kinase inhibitors: structural insights from biochemistry. Chem. Biol. 17, 1241-1249 (2010).

57. Kabsch, W. Xds. Acta Crystallogr. D 66, 125-132 (2010).

58. Winn, M. D. et al. Overview of the CCP4 suite and current developments. Acta Crystallogr. D 67, 235-242 (2011).

59. McCoy, A. J. et al. Phaser crystallographic software. J. Appl. Crystallogr. 40, 658-674 (2007)
60. Langer, G., Cohen, S. X., Lamzin, V. S. \& Perrakis, A. Automated macromolecular model building for X-ray crystallography using ARP/wARP version 7. Nat. Protoc. 3, 1171-1179 (2008).

61. Emsley, P. \& Cowtan, K. Coot: model-building tools for molecular graphics. Acta Crystallogr. D 60, 2126-2132 (2004).

62. Lebedev, A. A. et al. JLigand: a graphical tool for the CCP4 template-restraint library. Acta Crystallogr. D 68, 431-440 (2012).

63. Adams, P. D. et al. PHENIX: a comprehensive Python-based system for macromolecular structure solution. Acta Crystallogr. D 66, 213-221 (2010).

64. Chen, V. B. et al. MolProbity: all-atom structure validation for macromolecular crystallography. Acta Crystallogr. D 66, 12-21 (2010).

65. Gola, S., Martin, R., Walther, A., Dunkler, A. \& Wendland, J. New modules for PCR-based gene targeting in Candida albicans: rapid and efficient gene targeting using 100 bp of flanking homology region. Yeast 20, 1339-1347 (2003).

66. Gari, E., Piedrafita, L., Aldea, M. \& Herrero, E. A set of vectors with a tetracycline-regulatable promoter system for modulated gene expression in Saccharomyces cerevisiae. Yeast 13, 837-848 (1997).

67. Walther, A. \& Wendland, J. An improved transformation protocol for the human fungal pathogen Candida albicans. Curr. Genet. 42, 339-343 (2003).

68. Spellberg, B., Ibrahim, A. S., Edwards, Jr J. E. \& Filler, S. G. Mice with disseminated candidiasis die of progressive sepsis. J. Infect. Dis. 192, 336-343 (2005).

69. LaFayette, S. L. et al. PKC signaling regulates drug resistance of the fungal pathogen Candida albicans via circuitry comprised of $\mathrm{Mkc1}$, calcineurin, and Hsp90. PLoS Pathog. 6, e1001069 (2010).

70. Flynn, E. M. et al. A subset of human bromodomains recognizes butyryllysine and crotonyllysine histone peptide modifications. Structure 23, 1801-1814 (2015).

\section{Acknowledgements}

This work used the platforms of the Grenoble Instruct Center (ISBG: UMS 3518 CNRS CEA-UJF-EMBL) with support from FRISBI (ANR-10-INSB-05-02) and GRAL (ANR10-LABX-49-01) within the Grenoble Partnership for Structural Biology. We acknowledge the European Synchrotron Radiation Facility for provision of synchrotron radiation facilities and thank EMBL and ESRF staff for assistance at beamlines ID23-2, ID29 and ID30A-1, particularly M. Bowler and D. de Sanctis. We thank Myriam Ferro and Christophe Bruley for their general support, Sandrine Miesch-Fremy and Marie Arlotto for technical support, Joanna Timmins for access to and help with the CLARIOstar plate reader, Inah Kang for administrative support and EDyP team members for scientific discussions. This work was supported by grants from the FACE foundation (Partner University Fund to C.E.M. and C.P.), the National Institutes of Health (1R21AI113704 to C.E.M.), the Agence Nationale de Recherche (ANR-14-CE16-0027-01 (FungiBET) to C.P., J.G. and M.Co.; ANR-11-PDOC-011-01 (EpiGam) to J.G.; ANR-10-INBS-08 (ProFI) to J.G.; ANR-10-LABX-62-IBEID to C.D.), the EU FP7 Marie Curie Action (Career Integration Grant 304003 to J.G.) as well as by the USC Dornsife College of Letters, Arts and Sciences (E.F. and C.E.M.), a Chateaubriand Fellowship (E.F.) and a FINOVI fellowship from the Région Rhône Alpes, France (M.Cham.)

\section{Author contributions}

F.M., C.E.M., J.G. and C.P. conceived and designed the study. F.M., E.F., M.Cham., N.Z. D.M., Y.Z., M.Ha., D.S., C.G., M.Cou., M.Chau., C.D., B.A.K., M.Hu., M.Cor., J.G. and C.P. performed experiments and analysed data. C.E.M., J.G. and C.P. wrote the manuscript. All authors discussed results and commented on the manuscript.

\section{Additional information}

Supplementary Information accompanies this paper at http://www.nature.com/ naturecommunications

Competing interests: The authors declare no competing financial interests.

Reprints and permission information is available online at http://npg.nature.com/ reprintsandpermissions/

How to cite this article: Mietton, F. et al. Selective BET bromodomain inhibition as an antifungal therapeutic strategy. Nat. Commun. 8, 15482 doi: 10.1038/ncomms15482 (2017).

Publisher's note: Springer Nature remains neutral with regard to jurisdictional claims in published maps and institutional affiliations.

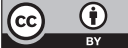

This work is licensed under a Creative Commons Attribution 4.0 International License. The images or other third party material in this article are included in the article's Creative Commons license, unless indicated otherwise in the credit line; if the material is not included under the Creative Commons license, users will need to obtain permission from the license holder to reproduce the material To view a copy of this license, visit http://creativecommons.org/licenses/by/4.0/

(C) The Author(s) 2017 